Review

\title{
An Overview of CMOS Photodetectors Utilizing Current-Assistance for Swift and Efficient Photo-Carrier Detection
}

\author{
Gobinath Jegannathan ${ }^{1, *(\mathbb{D})}$, Volodymyr Seliuchenko ${ }^{1,2}$, Thomas Van den Dries ${ }^{1}$ (D), Thomas Lapauw ${ }^{1}$ (D), \\ Sven Boulanger ${ }^{1}$, Hans Ingelberts ${ }^{1} \mathbb{D}$ and Maarten Kuijk ${ }^{1} \mathbb{D}$ \\ 1 Department of Electronics and Informatics (ETRO), Vrije Universiteit Brussel, 1050 Brussels, Belgium; \\ vse@melexis.com (V.S.); Thomas.Van.Den.Dries@vub.be (T.V.d.D.); Thomas.Lapauw@vub.be (T.L.); \\ sven.boulanger@vub.be (S.B.); hans.ingelberts@vub.be (H.I.); mkuijk@vub.be (M.K.) \\ 2 Melexis, Transportstraat 1, 3980 Tessenderlo, Belgium \\ * Correspondence: gobinath.jegannathan@vub.be
}

\section{check for} updates

Citation: Jegannathan, G.; Seliuchenko, V.; Van den Dries, T.; Lapauw, T.; Boulanger, S.; Ingelberts, H.; Kuijk, M. An Overview of CMOS Photodetectors Utilizing CurrentAssistance for Swift and Efficient Photo-Carrier Detection. Sensors 2021, 21, 4576. https://doi.org/10.3390/ s21134576

Academic Editor: Kevin Gallacher

Received: 18 May 2021

Accepted: 30 June 2021

Published: 4 July 2021

Corrected: 29 March 2022

Publisher's Note: MDPI stays neutral with regard to jurisdictional claims in published maps and institutional affiliations.

Copyright: (c) 2021 by the authors. Licensee MDPI, Basel, Switzerland. This article is an open access article distributed under the terms and conditions of the Creative Commons Attribution (CC BY) license (https:// creativecommons.org/licenses/by/ $4.0 /)$.

\begin{abstract}
This review paper presents an assortment of research on a family of photodetectors which use the same base mechanism, current assistance, for the operation. Current assistance is used to create a drift field in the semiconductor, more specifically silicon, in order to improve the bandwidth and the quantum efficiency. Based on the detector and application, the drift field can be static or modulated. Applications include 3D imaging (both direct and indirect time-of-flight), optical receivers and fluorescence lifetime imaging. This work discusses the current-assistance principle, the various photodetectors using this principle and a comparison is made with other state-of-the-art photodetectors used for the same application.
\end{abstract}

Keywords: current-assistance; CAPD; CMOS; fast time-gated cameras; demodulation; avalanche; Geiger

\section{Introduction}

Over the years, the prefix "current-assistance" has been found in an increasing number of different photonic sensors: current-assisted photonic demodulators (CAPD) for timeof-flight imaging [1-18], fast time-gated current-assisted photonic samplers (CAPS) for high-precision fluorescence lifetime imaging [19-22], current-assisted photodetector (CAP) for optical receivers [23] and current-assisted single-photon avalanche diodes (CA-SPAD) for fast and efficient single-photon detection $[24,25]$. This review paper focusses on research performed at the Department of electronics and informatics (ETRO), Vrije Universiteit Brussel (VUB) using this "current-assistance" principle.

\section{Current-Assistance Principle}

In Silicon photodetectors, it is always desirable to have a high quantum efficiency in the spectral region of interest. For applications requiring high quantum efficiency in the near infra-red (NIR) region, a quick solution is to have a thick/deep absorption layer because more low energy (or long wavelength) photons can be absorbed. The relation between the absorption and the epilayer thickness can be seen in Figure 1a,b. Unfortunately, there are no free rides on the Quantum Efficiency Express. A large photon-absorption volume will indeed result in more photo-generated carriers, but without any electric field present in the absorption volume, the main carrier transport mechanism is diffusion. Diffusion is where the free carriers move from a region of high carrier concentration to a region of low carrier concentration [26]. Diffusion is not directional and does not always lead to free carriers ending up in the region of interest, i.e., the detection node. Moreover, diffusion is a slower process compared to drift, thus the generated photo-carriers have a higher chance of recombination leading to a loss in quantum efficiency. In short, the pixel 
volume is "self-limiting" because if there is no electric field in the whole volume; this leads to drop in speed and in quantum efficiency.

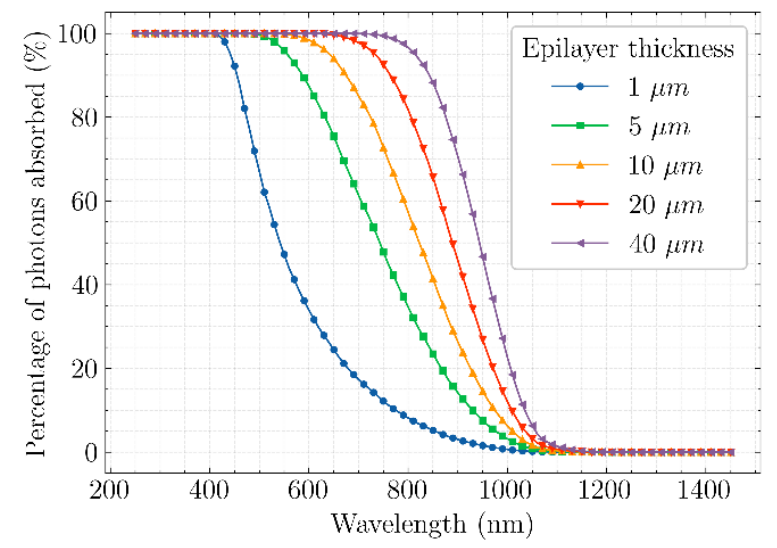

(a)

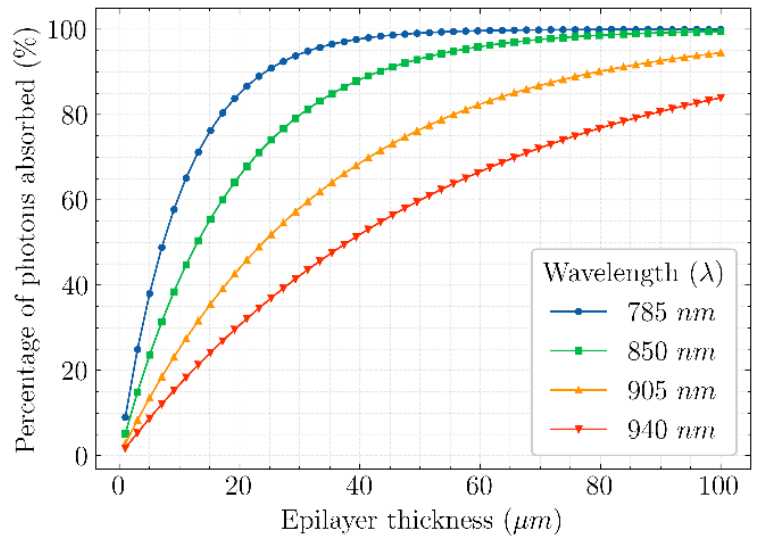

(b)

Figure 1. Absorption percentage in Silicon as a function of wavelength for different epilayer thicknesses (a) and absorption percentage in silicon as a function of epilayer thickness for a few wavelengths (b) (Absorption coefficient data from [27]).

The other major carrier transport mechanism is drift. Due to an electric field, carriers move swiftly with a drift velocity $\left(\mathrm{v}_{\mathrm{d}}\right)$. At low electric fields, the drift velocity follows the Equation (1), where it is directly proportional to the applied electric field (E) and the mobility of the carriers $(\mu)$. Here, the movement is faster compared to diffusion and very directional. Therefore, the generated photo-carriers can be directed, with the required speed, to the region of interest where they can be detected. Usually, the drift field is fast enough that almost all generated carriers can be detected before recombining. Recombination lifetimes $(\tau)$ range from nanoseconds to milliseconds depending on the dopant concentration and recombination-centers concentration [28].

$$
\mathrm{v}_{\mathrm{d}}=\mu \times \mathrm{E}
$$

Traditionally, a $p-n$ junction is used for photo-detection because of the built-in electric drift field that separates the generated electron-hole pairs. The electric drift field increases carrier speeds proportionally to the electric field strength (Equation (1)) up to carrier saturation velocity.

However, another way of establishing a drift field is through the application of a current through or voltage across a semiconductor material. Like with Ohm's law, it is hard to tell whether one applies a voltage and gets a current in return or vice versa. The resulting current-assisted drift field distribution, is different from the built-in (and/or applied) drift field distribution in the case of a $p$ - $n$ junction. Since the unavoidable assisting current makes the distinction between the two origins of having a drift field, the qualifier "current assistance" has been used systematically to distinguish detectors based on this type of drift-field. The drift field due to current-assistance also follows Equation (1) up to carrier saturation velocity.

As the origin of both types of electric field distribution are different, the possibilities, limitations, and challenges are different in each case. The current-assisted drift fields can be switched in direction, and in some cases allow more easily to fill a larger semiconductor volume with a drift field. However, the cost of applying this drift field is the current-flow between the two regions, due to a flow of majority carriers (holes in $p$-type semiconductor and electrons in $n$-type semiconductor) and its associated power dissipation.

For example, in a $p-i-p$ semiconductor structure, when a potential difference is applied between the two $p$-type regions, there will be a flow of holes from the $p$-type region with a higher potential to the $p$-type region with a lower potential. Now, if a photon is absorbed in the $i$-region, it will generate an electron-hole pair. The photoelectron will experience a 
drift field towards the $p$-type region with the higher potential while the associated hole will drift in the opposite direction. This phenomenon is illustrated in Figure 2.

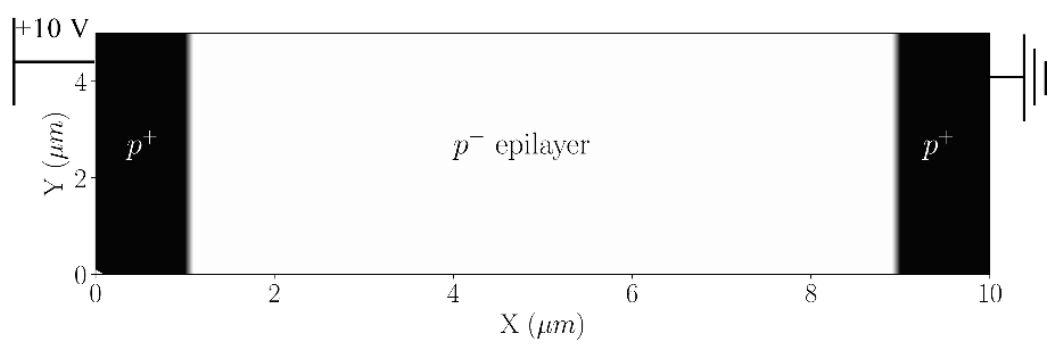

(a)

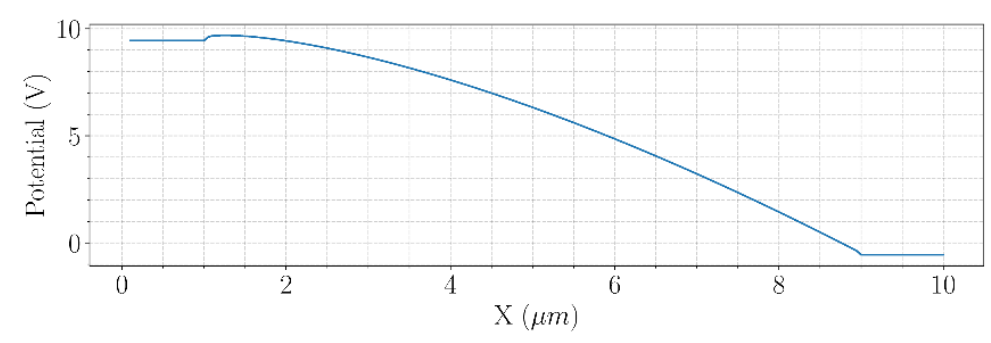

(b)

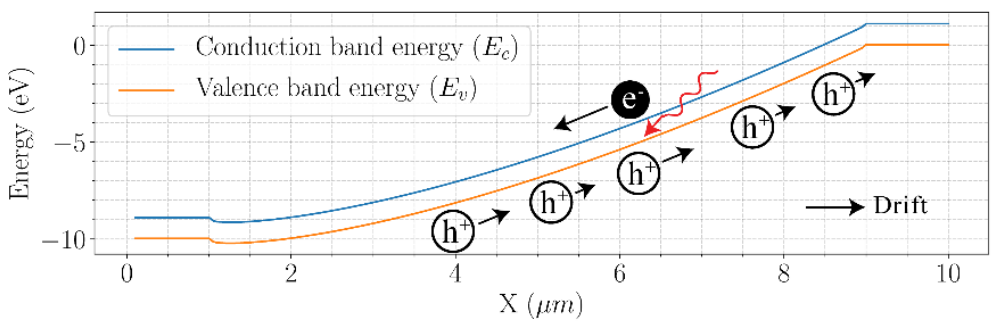

(c)

Figure 2. Cross-section of a simple p-i-p structure (a), the potential as a function of $\mathrm{X}$ (at any point in $Y)(b)$ and the corresponding conduction and valence band energies with overlaid illustration of current-assistance operation (c).

The current assistance principle is illustrated in Figure 3 by a planar CMOS technology compatible structure and is simulated in Silvaco Atlas device physics simulator [29]. This cylindrical photodetector, situated in a high-resistivity $p^{-}$epilayer $(\sim 1000 \Omega \cdot \mathrm{cm})$, has a very small $p-n$ junction diode in the center. The diode comprises of a $\mathrm{n}^{+}$cathode surrounded by a $\mathrm{p}^{+}$annular anode. This "central" photodiode is surrounded by a larger ring of $\mathrm{p}^{+}$ doping, which we will refer to as ring. The extent of the ring marks the boundaries of the photodetector and the area encapsulated by the ring is the photo-absorption area. The central $p-n$ junction is reverse biased, and the ring is biased at a lower potential than the anode. Due to this potential difference, there is a hole current between the anode and the ring. The photo-generated electrons in the volume between the anode and the ring will be swiftly guided towards the anode with a drift velocity $\left(v_{d}\right)$. When the photo-electrons reach the anode, they only have to diffuse a very small distance to fall into the depletion region and get detected by the cathode. There is a depletion region in the epilayer, whose area is proportional to the reverse bias voltage and the doping concentration, that normally does not extend beyond the epilayer due to the high doping levels of the $\mathrm{p}^{+}$substrate. 


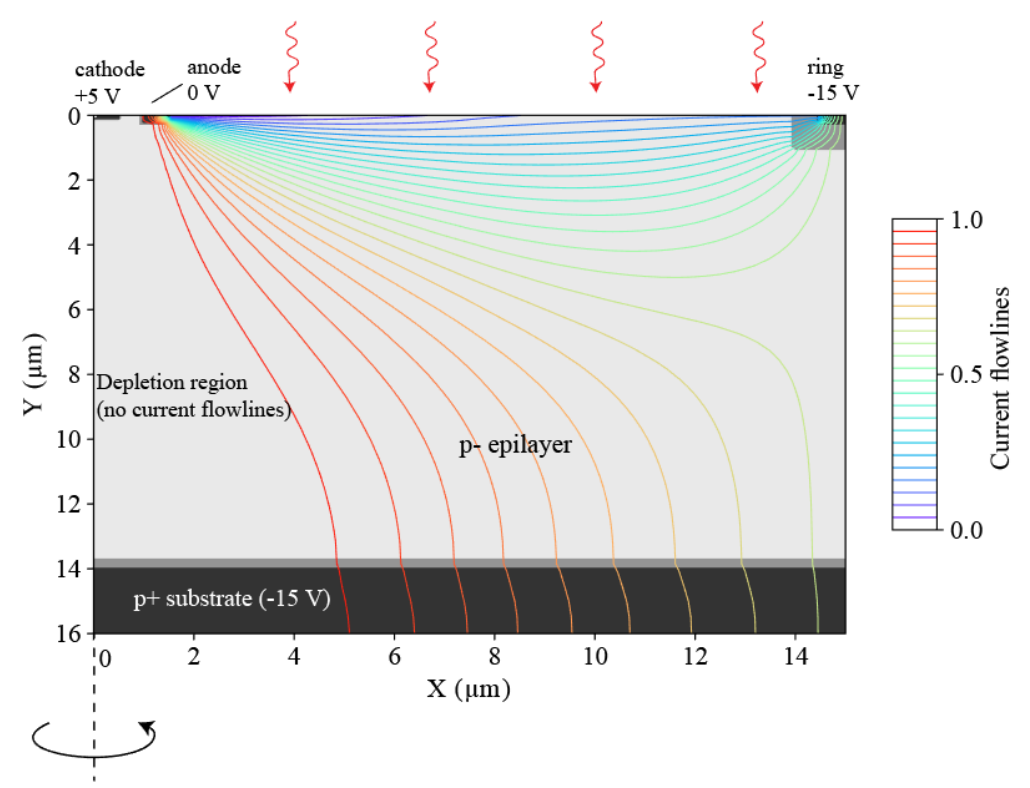

Figure 3. Cross section of the example structure to demonstrate current-assistance principle with an overlay of simulated current flowlines.

Once the photoelectrons reach the vicinity of the anode region, they will have to diffuse a small distance, due to a small potential gradient around the anode, to fall into the depletion region between the anode and the cathode at which point they are detected. The photo-detection bandwidth is dictated by the size of the detector, the larger the detector the more the transit time to reach the detection area. In practice, diffusion of the photoelectrons near the anode can be a main factor which limits the bandwidth of current-assisted detectors with a lateral drift field. A simulated impulse response of the example detector, when illuminated uniformly with a sharp (10 ps), $850 \mathrm{~nm}$, is shown for different ring voltages (cathode-anode voltage fixed at $+5 \mathrm{~V}$ ) in Figure 4 . When the ring voltage is the same as the anode voltage, then there is no drift field and diffusion becomes the only transport mechanism, leading to only few photoelectrons reaching the cathode (the rest recombine) and thereby reducing the quantum efficiency. With increasing ring voltages $(-5 \mathrm{~V}$ to $-30 \mathrm{~V})$; however, the impulse response keeps getting sharper and converges to a maximum due to velocity saturation. This structure is very similar to that of the current-assisted photodiode (CAP) which will be explained in more detail in the next section (Section 3.1).

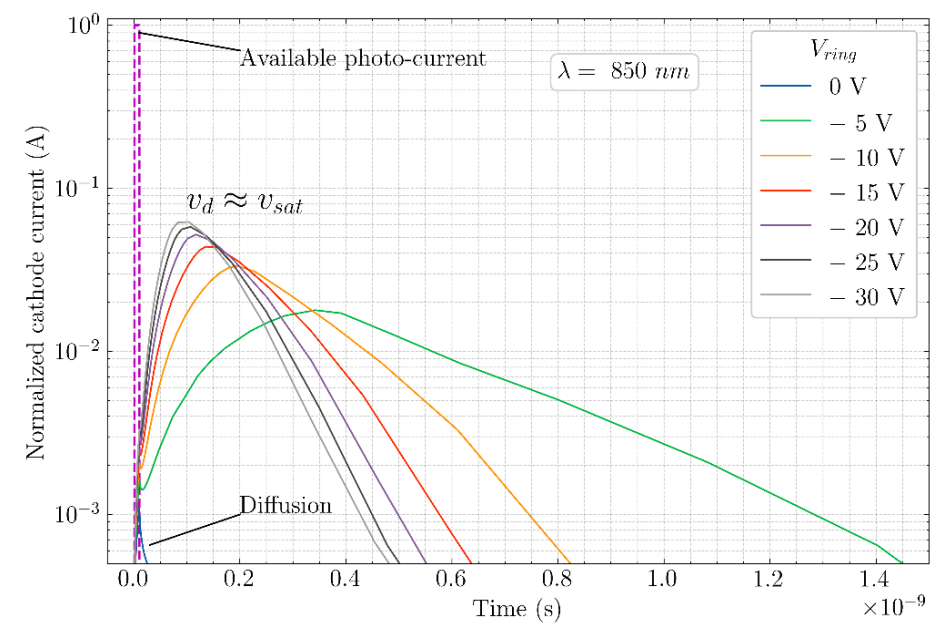

Figure 4. Simulated timing response of the device when illuminated with a sharp light pulse (10 ps) of $850 \mathrm{~nm}$ for a few different ring voltages. 


\section{CMOS Photodetectors Using Current-Assistance}

\subsection{Current-Assisted Photodetector (CAP)}

At the basis of the majority current modulation lies the $p$-i- $p$ structure, which has been shown to exhibit photogain through accumulation or trapping of minority carriers [30-32]. Therefore, it is recognized that the assisting majority current itself is modulated significantly by the incident light, and thus can be included to the total AC detection current. The $p-i-p$ structure allows trapping of electrons inside the substrate. This helps the accumulation of holes in the substrate, which in turn reduces the overall resistivity of the detector. In general, the high-low junction barrier height, as well as the thickness of the barrier $\mathrm{p}^{+}$ region (see Figure 5) determines how well the electrons can be trapped. This trapping of minority carriers is known to improve responsivity dramatically, but it at the same time reduces the bandwidth just as much. This behavior is also known to be very nonlinear with respect to the incident optical power.

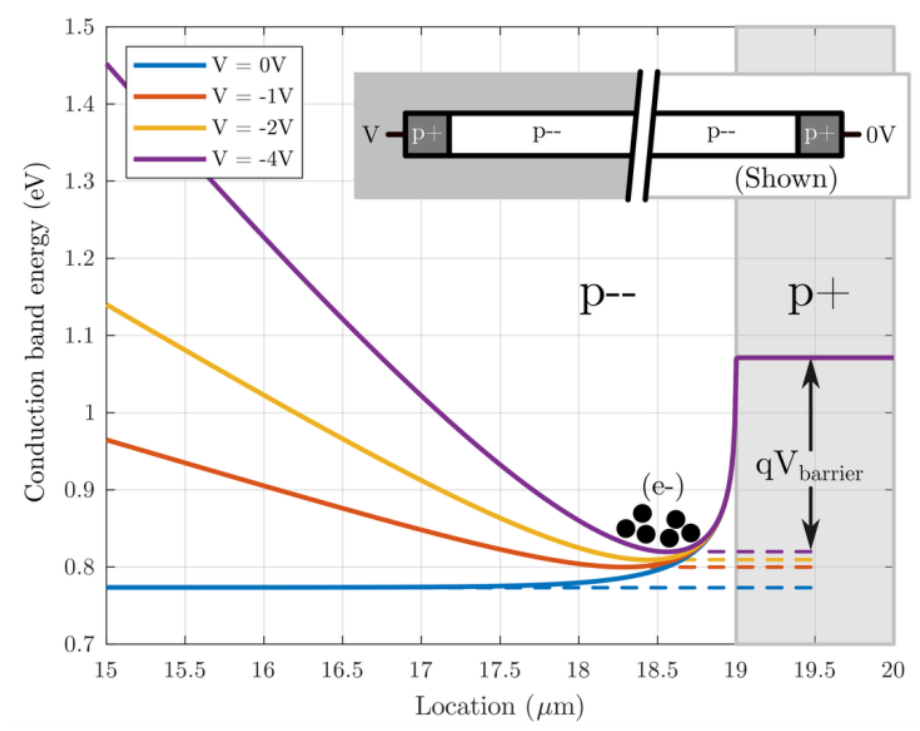

Figure 5. The simulated conduction band of a $p-i-p$ photodetector shows how photogenerated minority charge carriers can be trapped and the cross-section of the $p$-i- $p$ detector (inset).

The simplest photodetector that makes use of current-assistance is the CurrentAssisted Photodiode (CAP) [32]. Rather than trapping all electrons inside the substrate, the CAP provides a sink for photoelectrons by means of the cathode $\mathrm{n}^{+}$region. This structure is shown in Figure 6. This means that the bandwidth will be improved at the cost of photo-gain. Like with any "current-assisted" detector, we can identify three travel modes for minority carriers determining the overall bandwidth.

Initially, most minority carriers will be generated inside the substrate. At this point, a drift field is needed to bring them to the anode, often resulting in the need to apply a backside potential as well. Along the anode edge, they accumulate and diffuse into the cathode, where they can drift again once they reach the depletion region. A worst-case, average time constant for these situations can be modeled.

$$
\begin{gathered}
\tau_{\mathrm{dr}}=\frac{1_{\text {drift }}}{\mu|\vec{E}|} \\
\tau_{\text {diff }}=\frac{1_{\mathrm{d}}^{2}}{2 \mathrm{n}_{\mathrm{dim}} \mathrm{D}_{\mathrm{n}}} \\
\tau_{\text {tot }}^{2}=\tau_{\mathrm{dr} 1}^{2}+\tau_{\mathrm{diff}}^{2}+\tau_{\mathrm{dr} 3}^{2}
\end{gathered}
$$




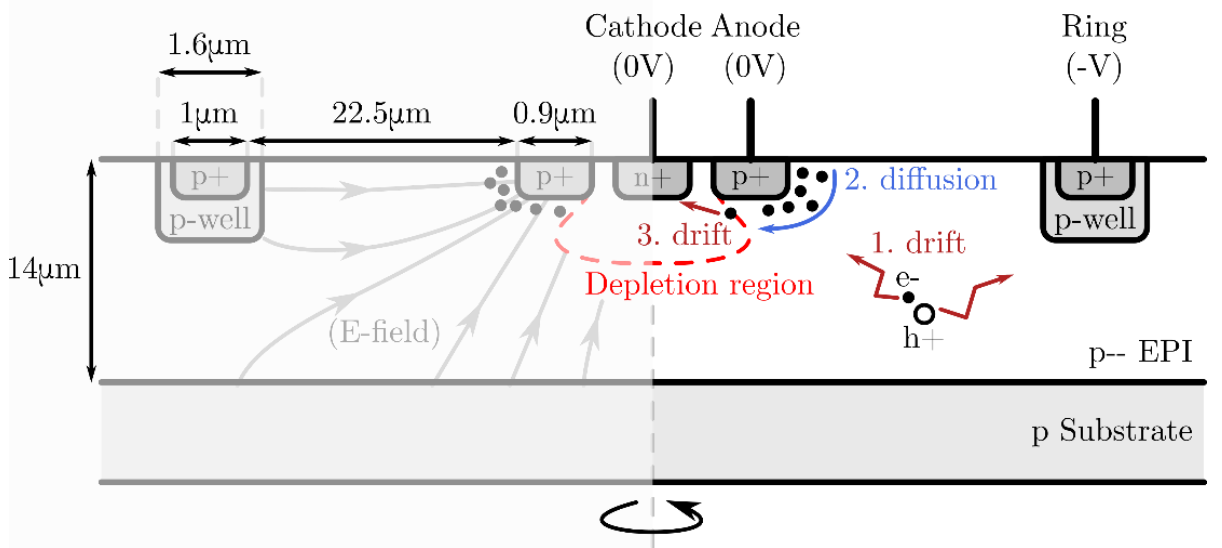

Figure 6. The layout and dimensions of the fabricated current-assisted photodiode. The center cathode $\mathrm{n}^{+}$region is $1.5 \mu \mathrm{m}$ in diameter, keeping $0.75 \mu \mathrm{m}$ space with the anode $\mathrm{p}^{+}$region. The ring is biased to a negative bias voltage.

Here, $1_{\text {drift }}$ is the average distance traveled while in the strong drift field, while $\mu$ is the minority carrier mobility and $|\vec{E}|$ the electric field strength. $1_{d}$ is the average distance traveled along the edge of the anode $\mathrm{p}^{+}$-region, $\mathrm{n}_{\text {dim }}$ represents the number of dimensions, and $D_{n}$ is the minority-carrier diffusion constant. Usually, the combination of $\tau_{\text {diff }}$, the average time spent diffusing, and $\tau_{\mathrm{dr} 1}$, the average time spent drifting in the bulk towards the anode, decides the detection bandwidth [33]. The last drift stage (the third travel mode, $\left.\tau_{\mathrm{dr} 3}\right)$ where the charge carrier is captured by the depletion region near the center cathode is often negligible. For small pixel pitches $(<10 \mu \mathrm{m}), \tau_{\text {diff }}$ plays a more important role in limiting bandwidth and for large pixel pitches $(>100 \mu \mathrm{m}) \tau_{\mathrm{dr} 1}$ can become large enough to limit bandwidth. The minority carrier transport time is dependent on the location of the photogenerated minority carriers within the device, e.g., the minority carriers that are generated directly below the cathode $\mathrm{n}^{+}$region drift directly towards that $\mathrm{n}^{+}$region without diffusion along the anode $\mathrm{p}^{+}$region. The expected value for the total time for a minority carrier to reach the cathode (Equation (4)) is therefore a worst-case scenario.

Figure 7 illustrates the photo-gain bandwidth tradeoff as a function of the anode region $\left(\mathrm{p}^{+}\right)$width. We keep $V_{\text {cathode }}=V_{\text {anode }}=0 \mathrm{~V}$, and have $V_{\text {ring }}=V_{\text {substrate }}=-3 \mathrm{~V}$. The simulated structure is cylindrically symmetric with a ring diameter of $50 \mu \mathrm{m}$.

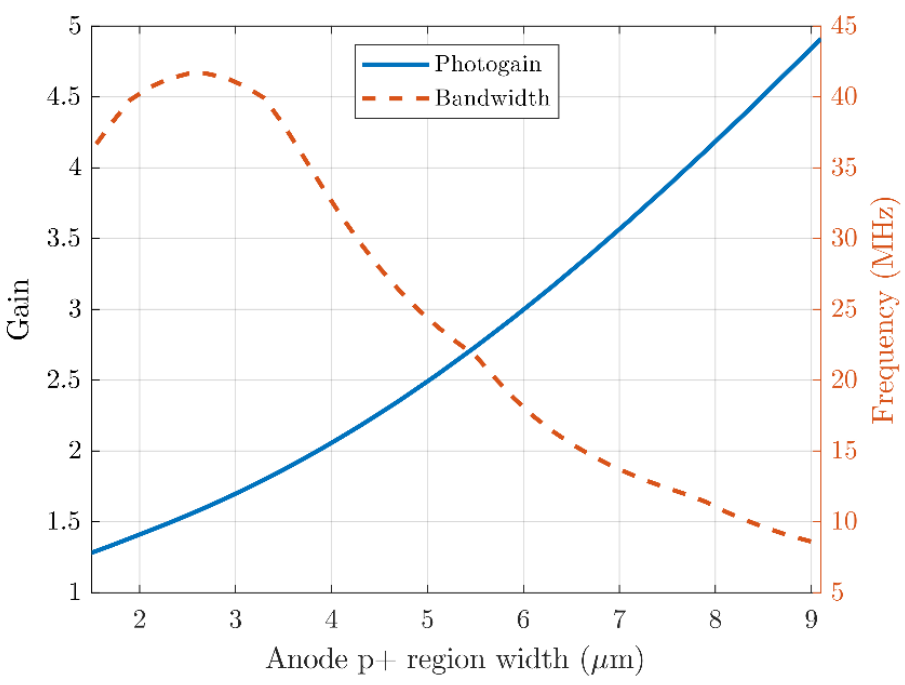

Figure 7. The simulated photo-gain and bandwidth for varying anode $\mathrm{p}^{+}$region cross-section widths. The width can be used to trade bandwidth for photo-gain. 
For small $\mathrm{p}^{+}$regions, the uniformity of the electric field inside the epitaxial layer determines the bandwidth. For larger $\mathrm{p}^{+}$regions, we find that the accumulation of photogenerated electrons near the anode $\mathrm{p}^{+}$region causes both an increase in photo-gain, as well as a decrease in bandwidth. For larger $\mathrm{p}^{+}$regions, the DC biasing current also increases which may not be desirable.

The measured responsivity shows that photo-gain is indeed feasible (shown in Figure 8). The cathode current responsivity is quasi constant over incident optical power, reinforcing the fact that all minority carriers are ultimately collected by the cathode $\mathrm{n}^{+}$ region. However, we see that the anode current responsivity also has a very significant contribution towards the total photocurrent, especially at low light intensities as supported by simulations.

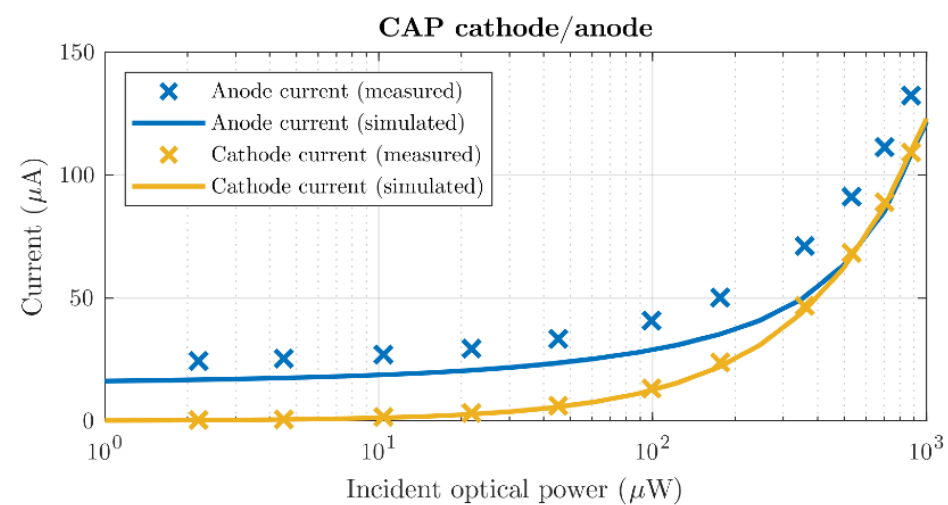

(a)

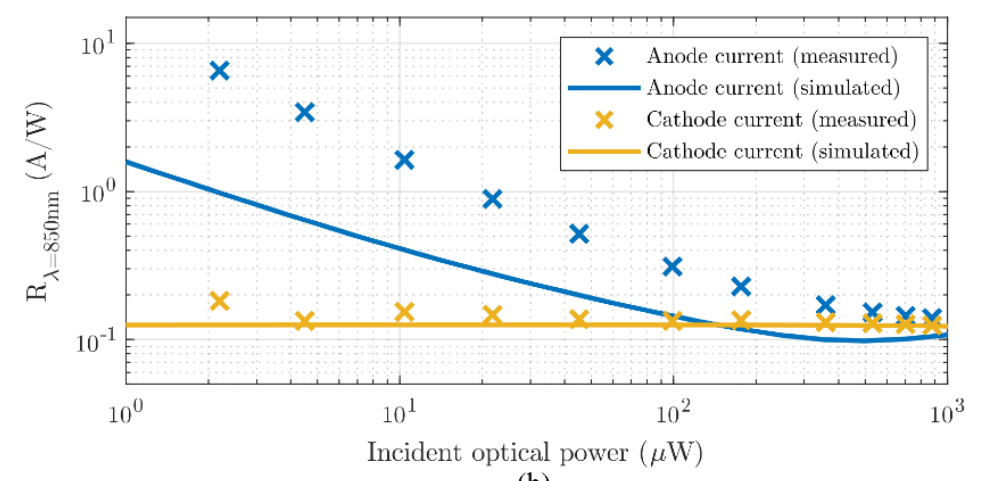

(b)

Figure 8. Measured and simulated anode and cathode current (a) and responsivity (b). $\mathrm{V}_{\text {anode }}=\mathrm{V}_{\text {cathode }}=0 \mathrm{~V}, \mathrm{~V}_{\text {ring }}=\mathrm{V}_{\text {substrate }}=-5 \mathrm{~V}$.

The discrepancies between simulated and measured anode current can be traced to incomplete modeling of the junctions. Simulations show sensitivity to substrate doping levels, junction depths and surface trap state densities.

The majority current contribution can also be significant at higher frequencies, as shown in Figure 9. It was measured by exciting an OPV300 VCSEL (Optek Technology, Carrollton, TX, USA) $(\lambda=850 \mathrm{~nm})$ using a PRBS7 signal at multiple sampling frequencies. The sampled signal was analyzed at the excited frequency bins after windowing to reduce spectral leakage. The signals were measured using an Agilent Infinium DCA-J 86100C (Agilent Technologies, Santa Clara, CA, USA) with an 86117A $30 \mathrm{GHz}$ channels module where the signals were terminated by a $50 \Omega$ load resistance. The "true" optical input signal was simultaneously measured using a Thorlabs DXM12DF DC $-12 \mathrm{GHz}$ (Thorlabs, Inc., Newton, NJ, USA) detector using a beam splitter. 


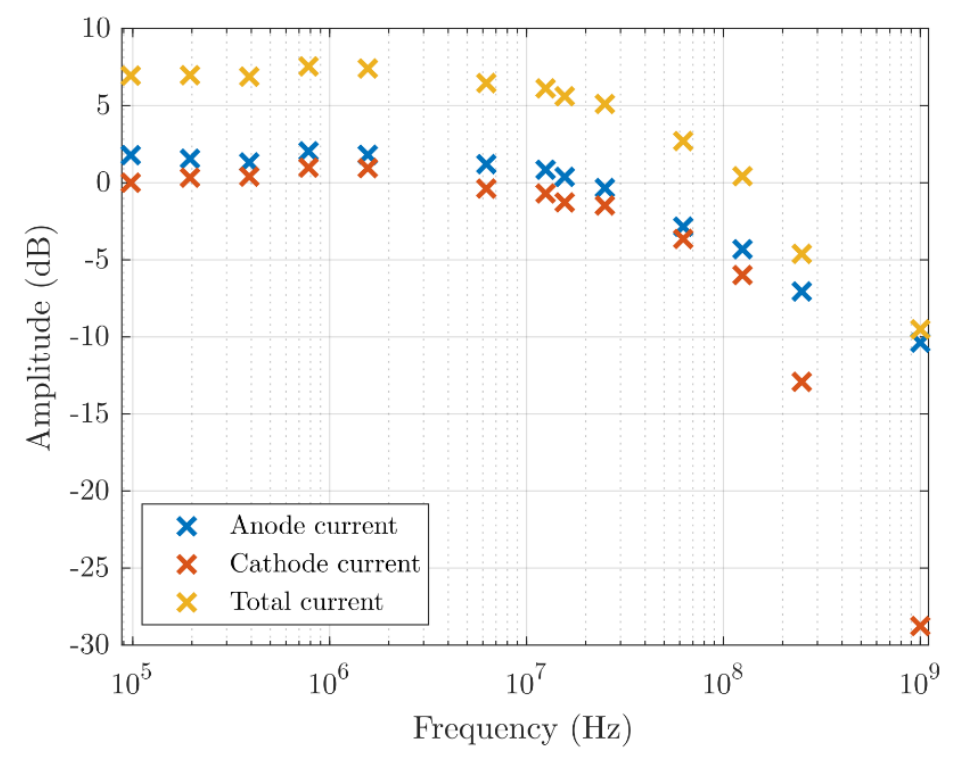

Figure 9. The normalized frequency response of the anode and cathode current at an average optical power of $360 \mu \mathrm{W}, \mathrm{V}_{\text {anode }}=\mathrm{V}_{\text {cathode }}=0 \mathrm{~V}, \mathrm{~V}_{\text {ring }}=\mathrm{V}_{\text {substrate }}=-5 \mathrm{~V}$.

The measured frequency response shows that the anode current supports similar frequencies as the cathode current. To reach higher bandwidths with this type of detector, a viable option is to combine both the cathode and anode currents into one photocurrent. The result is a small photo-gain and an extended bandwidth through accumulation of minority carriers [30-32].

For noise, we can expect two dominant noise sources: shot noise is expected for minority current, while thermal noise is expected for the majority current. Since the minority current (and its shot noise) directly modulates the majority current (and so the generated thermal noise), a more involved approach is needed to model the overall noise behavior. Noise performance has not been measured and is subject to further research.

\subsection{Current-Assisted Photonic Demodulator (CAPD)}

The current-assistance principle allows to modulate the electrical drift field distribution through modulation of the voltage sources connected to the substrate ohmic contacts. This concept is employed in the current-assisted photonic demodulator (CAPD) devices that have been successfully used in numerous indirect TOF (iTOF) distance sensing applications.

The CAPD device was described in detail in van Nieuwenhove et al. [12], and van der Tempel [33]. The general idea of the CAPD is illustrated in Figure 10. In this example, the photodetector is built in the lowly doped p-type substrate designated with a "Si epi" label. The CAPD is composed of a substrate resistor, formed between the two $\mathrm{p}^{+}$regions (labelled " $\mathrm{P}$ "), and the two $\mathrm{n}^{+}$regions (labelled " $\mathrm{N}^{\prime}$ ) located in the close proximity to the $\mathrm{p}^{+}$regions. When a voltage source Vmix is connected to these $\mathrm{p}^{+}$regions, a majority hole current will flow through the substrate resistor creating a gradual voltage drop and, therefore, an electric field in the silicon substrate. Light, incident on this device from the top, is absorbed in the substrate creating electron-hole pairs. The photo-electrons are drifting along the electric field lines towards the $p$ region with a higher potential creating a pool of electrons around the $\mathrm{p}^{+}$-region. These photoelectrons cannot penetrate the $\mathrm{p}^{+}$-region because of a potential barrier stemming from the doping difference between the highly-doped $\mathrm{p}^{+}$-region and a lowly-doped $\mathrm{p}^{-}$-substrate. This electron pool is drained by placing an $\mathrm{n}^{+}$doped region near $\mathrm{p}^{+}$-doped region. Therefore, the minority carrier electron current stemming from photo generation can be measured separately from the majority hole electrical current which assists in creating an electrical drift field in the substrate. Because of such separation, there is no extra thermal or shot noise contribution from the majority substrate current. 


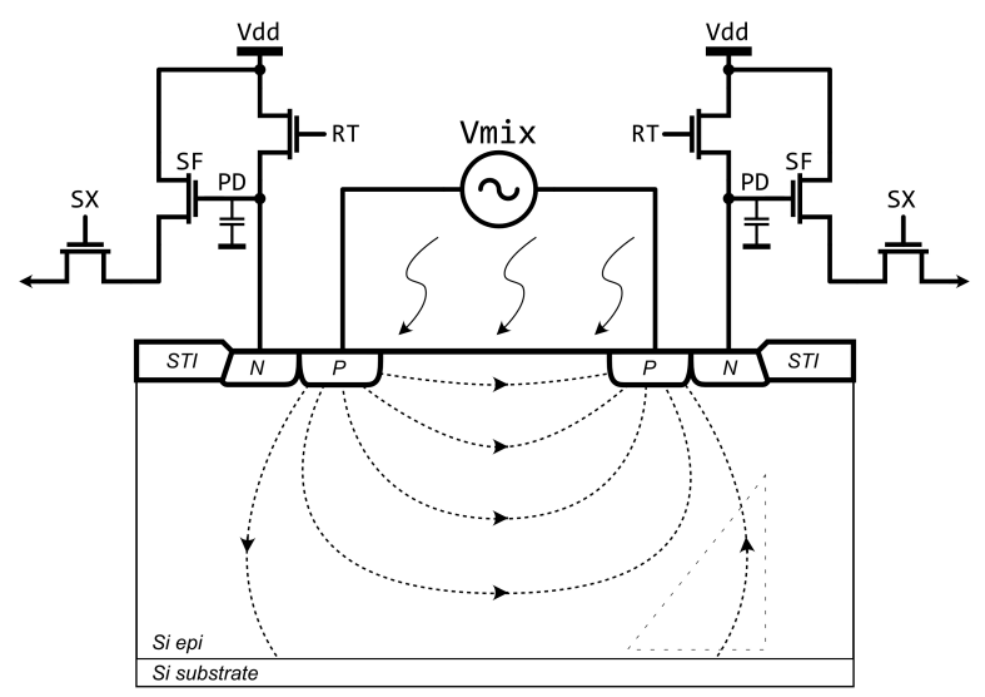

Figure 10. 3-transistor (3T) 2 tap iTOF CMOS pixel architecture.

When an alternating voltage is applied to the $\mathrm{p}^{+}$-regions, the photo electron current can be guided either to one $\mathrm{n}^{+}$- region or the other, thus, an electronic shutter for the optical signal, controlled by the voltage source Vmix, is formed.

The photo-electrons accumulate in the $\mathrm{n}^{+}$doped regions and the signal can be read out using circuits that are typically used in the conventional 3-transistor (3T) and 4-transistor (4T) image pixels. Figure 10 shows an example with $3 \mathrm{~T}$ readout circuits connected to the 2 collecting $\mathrm{n}^{+}$doped regions (taps). The operation of the readout circuits is similar to the conventional 2D image sensor readout. Both PD nodes are reset with reset RT transistors at the start of the integration, and the photo-electrons are integrated on the PD nodes which accumulate in-phase and out-of-phase photo-electron signals. At the end of the integration the voltage signals are read out through the source follower transistors SF and row select switches SX. The CAPD pixels can also have more complex readout circuitry like $4 \mathrm{~T}$ readout circuits, sample and hold circuits for improved global shutter performance, circuits for dynamic range extension or common mode signal subtraction.

The iTOF principle is closely related to the lock-in signal detection technique developed in 1946 by R.H. Dicke to improve sensitivity of microwave radar receivers [34]. The lock-in technique allows signal recovery in noisy environments by advantageously exploiting prior knowledge about the signal itself. The lock-in correlation signal can have a higher signal to noise ratio (SNR) than the original signal since the signal is correlated to the reference signal, but the random noise is not, thus, enabling signal detection in noisy environments. CAPD devices can be used to measure the correlation signal between the optical signal and the reference electrical signal.

Although, an arbitrary electrical reference signal can be applied to the CAPD to modulate the drift field, a square wave signal is most often used in practice. A photonic demodulator can be modelled as an electronic shutter device which creates a temporal window rect $\mathrm{CAPD}_{\mathrm{A}}(\mathrm{t})$ for the impinging optical signal what is equivalent to multiplication of the square wave reference signal and the impinging optical signal. The signal within this temporal window gets integrated on the sensor's integration capacitor and the resulting voltage signal is then sampled. Mathematically this temporal windowing and integration processes can be described as a convolution or cross-correlation of the optical signal with a rectangular window function and sampling can be modelled by a Dirac comb function. Thus, the iTOF signal $s(t)$ can be represented as:

$$
\mathrm{s}(\mathrm{t})=\left[\mathrm{o}(\mathrm{t}) * \operatorname{rect}_{\mathrm{CAPD}}\left(\frac{\mathrm{t}}{\mathrm{t}_{\mathrm{pd}}}\right)\right] \times \sum_{\mathrm{n}=-\infty}^{+\infty} \delta\left(\mathrm{t}-\frac{\mathrm{n} \mathrm{T}_{\mathrm{mod}}}{\mathrm{m}}\right)
$$


where $\mathrm{o}(\mathrm{t})$ is the incoming detected optical signal, $\mathrm{t}_{\mathrm{pd}}$ is the temporal window width, $\mathrm{T}_{\text {mod }}$ is the modulation period, $\mathrm{m}$ is the number of phases, i.e., number of temporal windows per modulation period, and $\mathrm{n}$ is the integer sample number $\mathrm{n} \in \mathbb{Z}$. In a general case, at least three samples are required to recover the three unknown parameters in the incoming optical signal: amplitude, offset and phase shift. The most common iTOF sensor design with two photo-detector nodes (taps) creates two (in-phase and out-of-phase) temporal windows. However, such a two tap iTOF sensor can still be used to find the phase shift of the incoming optical signal: the necessary extra samples needed to find the three unknown parameters of the incoming light signal can be created by repeating the measurement with an extra phase shift introduced between the light signal and the CAPD electrical drift field modulation signal. The samples from multiple measurements may be combined producing a signal similar to the output signal from an iTOF sensor with a higher number of taps.

Figure 11a illustrates the sampled TOF signal from a four tap iTOF sensor. Each tap A, $\mathrm{B}, \mathrm{C}$ and $\mathrm{D}$ is sampling the signal integrated over its corresponding time window. These samples form a periodic time domain signal $\mathrm{s}_{0}, \mathrm{~s}_{1}, \mathrm{~s}_{2}, \mathrm{~s}_{3}$.

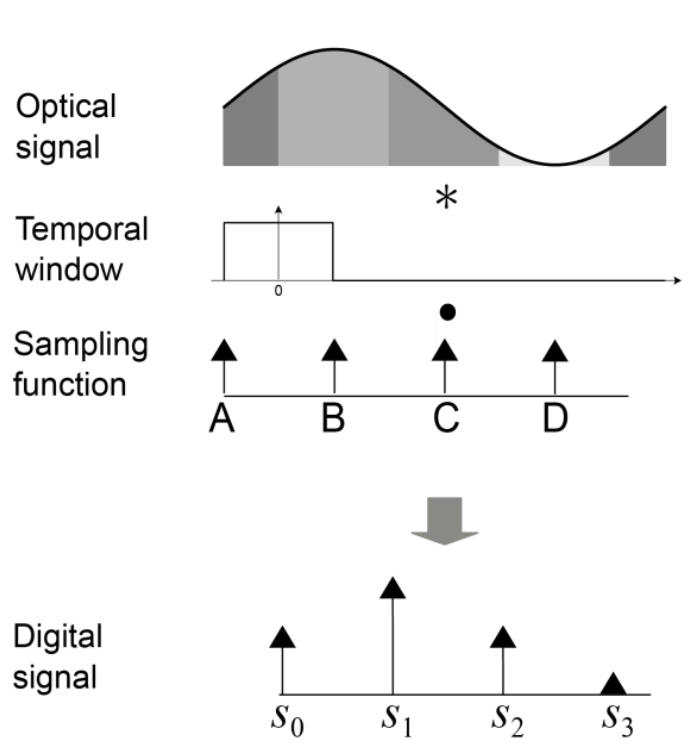

(a)
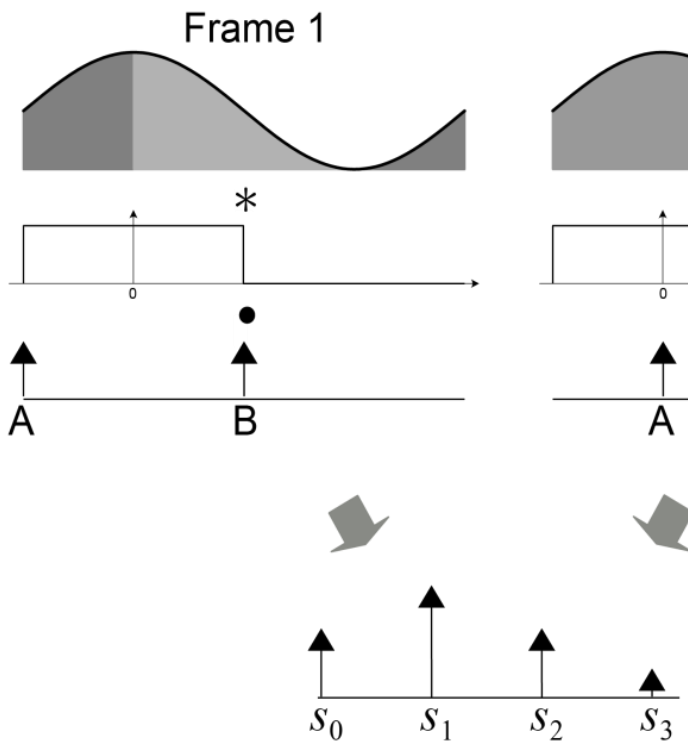

(b)

Figure 11. iTOF sampling for a 4 tap pixel (a) and a pseudo 4 tap pixel using 2 tap pixel (b). The * sign denotes the convolution in Equation (5).

Figure $11 b$ illustrates a signal from two measurements using a two tap iTOF sensor. Taps A and B are sampling the signal integrated over its corresponding time window for two consecutive frame acquisitions, the frames having pi/2 phase shift. The four samples acquired over two frames can be recombined to form a periodic time domain signal similar to $s_{0}, s_{1}, s_{2}, s_{3}$.

In practice, the gating and integration process is repeated many times during the integration time $\mathrm{T}_{\text {int }}$. For brevity, we will consider just one modulation cycle in the mathematical description of iTOF sensor operation.

After acquisition, the signal can be analyzed by means of a discrete Fourier transform (DFT). The first DFT complex valued bin contains information about the amplitude and the phase offset of the sampled iTOF signal's first harmonic from which the TOF distance can be calculated.

For an m phase iTOF sensor, the DFT coefficients can be expressed as:

$$
\mathrm{S}_{\mathrm{b}}=\sum_{\mathrm{k}=0}^{\mathrm{m}-1} \mathrm{~s}_{\mathrm{k}} \mathrm{e}^{-\mathrm{j} \theta_{\mathrm{k}} \mathrm{b}}=\sum_{\mathrm{k}=0}^{\mathrm{m}-1} \mathrm{~s}_{\mathrm{k}}\left[\cos \left(\theta_{\mathrm{k}} \mathrm{b}\right)-\mathrm{j} \sin \left(\theta_{\mathrm{k}} \mathrm{b}\right)\right]
$$


where $\theta_{k}=2 \pi \frac{k}{m}$, and $s_{k}$ is the $k$-th iTOF sample. The DFT complex valued bins can be represented using the real and imaginary components $\mathrm{I}_{\mathrm{b}}$ and $\mathrm{Q}_{\mathrm{b}}$ :

$$
S_{b}=I_{b}-j Q_{b}
$$

where $\mathrm{I}_{\mathrm{b}}=\sum_{\mathrm{k}=0}^{\mathrm{m}-1} \mathrm{~s}_{\mathrm{k}} \cos \left(\theta_{\mathrm{k}} \mathrm{b}\right)$, and $\mathrm{Q}_{\mathrm{b}}=\sum_{\mathrm{k}=0}^{\mathrm{m}-1} \mathrm{~s}_{\mathrm{k}} \sin \left(\theta_{\mathrm{k}} \mathrm{b}\right)$. The TOF phase shift and amplitude signals can be then calculated using the first DFT bin as:

$$
\begin{gathered}
\phi=\operatorname{atan} 2\left(\mathrm{Q}_{1}, \mathrm{I}_{1}\right) \\
\mathrm{A}=\sqrt{\mathrm{I}_{1}^{2}+\mathrm{Q}_{1}^{2}}
\end{gathered}
$$

For example, for the case of 4 tap iTOF pixel $(\mathrm{m}=4)$, the phase $\phi$ and amplitude A can be calculated as:

$$
\begin{gathered}
\mathrm{I}_{1}=\mathrm{s}_{0}-\mathrm{s}_{2}, \mathrm{Q}_{1}=\mathrm{s}_{1}-\mathrm{s}_{3} \\
\phi=\operatorname{atan} 2\left(\mathrm{~s}_{1}-\mathrm{s}_{3}, \mathrm{~s}_{0}-\mathrm{s}_{2}\right), \\
\mathrm{A}=\sqrt{\left(\mathrm{s}_{0}-\mathrm{s}_{2}\right)^{2}+\left(\mathrm{s}_{1}-\mathrm{s}_{3}\right)^{2}}
\end{gathered}
$$

The TOF distance to the object $d$ is:

$$
\mathrm{d}=\frac{\phi}{4 \pi} \times \mathrm{c} \times \mathrm{T}_{\mathrm{mod}}
$$

The second and higher order DFT bins can also be used to extract other useful iTOF signal indicators such as motion or saturation depth $[35,36]$. The iTOF distance error and SNR can be calculated as [6]:

$$
\begin{gathered}
\sigma_{\text {depth }} \equiv \frac{1}{\sqrt{2} \cdot \mathrm{SNR}} \times \frac{1}{2 \pi} \times \frac{\mathrm{c}}{2 \cdot \mathrm{F}_{\text {mod }}} \\
\mathrm{SNR} \equiv \frac{\mathrm{A}_{\text {pix }} \times \mathrm{R} \times \mathrm{FF} \times \mathrm{C}_{\text {mod }} \times \Phi_{\text {active }} \times \mathrm{t}_{\text {int }} \times \frac{1}{\mathrm{q}}}{\sqrt{\mathrm{A}_{\text {pix }} \times \mathrm{R} \times \mathrm{FF} \times\left(\Phi_{\text {active }}+\Phi_{\text {ambient }}\right) \times \mathrm{t}_{\text {int }} \times \frac{1}{\mathrm{q}}+\mathrm{N}_{\text {system }}^{2}}}
\end{gathered}
$$

where $\mathrm{A}_{\text {pix }}$ is the pixel area, $\mathrm{FF}$ - fill factor, $\mathrm{R}$-responsivity, $\mathrm{C}_{\bmod }$ - demodulation contrast, $\mathrm{F}_{\text {mod }}$ - modulation frequency, $\mathrm{t}_{\mathrm{int}}$-integration time, $\mathrm{N}_{\text {system }}^{2}$ - variance of the system noise floor, $\Phi_{\text {active }}$ is sensor irradiance due to the active illumination and $\Phi_{\text {ambient }}$ is the sensor irradiance due to the ambient illumination.

To achieve the lowest distance noise, the product $\left[\sqrt{A_{\text {pix }} \times R \times F F}\right] \times\left[C_{\text {mod }} \times F_{\text {mod }}\right]$ should be maximized. The first factor $\left[\sqrt{\mathrm{A}_{\text {pix }} \times \mathrm{R} \times \mathrm{FF}}\right]$ is defined by the optical to electrical signal conversion efficiency, and the second factor $\left[\mathrm{C}_{\bmod } \times \mathrm{F}_{\mathrm{mod}}\right]$ is defined by the CAPD speed.

The current assistance principle allows to create electric drift field across large semiconductor volumes to achieve high $A_{\text {pix }}, R$ and $F F$, thus maximizing $\left[\sqrt{A_{\text {pix }} \times R \times F F}\right]$.

The CAPD speed factor $\left[\mathrm{C}_{\text {mod }} \times \mathrm{F}_{\text {mod }}\right]$ can be estimated from solution of the continuity equations [26] that govern the transport of the photo electrons:

$$
\frac{\partial \mathrm{n}_{\mathrm{p}}}{\partial \mathrm{t}}=\mathrm{G}_{\mathrm{n}}-\mathrm{R}_{\mathrm{n}}+\nabla \times \mathrm{J}_{\mathrm{n}}+\mathrm{G}_{\mathrm{L}}
$$

where $n_{p}$ is the minority carrier concentration in the $p$ substrate, $G_{n}$ and $R_{n}$ are generation and recombination rates, $G_{L}$ is the generation rate due to impinging light signal, and $J_{n}$ is the electron current density:

$$
\mathrm{J}_{\mathrm{n}}=\mathrm{qn} \mu_{\mathrm{n}} \mathrm{E}_{\mathrm{drift}}+\mathrm{qD}_{\mathrm{n}} \nabla \mathrm{n}
$$


Detailed modelling of the photo currents is done in Estrada et al. [8]. An approximate estimation of the electron transient time across the length of the device can be done assuming linearly distributed electrical drift field across the device. For example, for a $15 \mu \mathrm{m}$ device biased by a $1 \mathrm{~V}$ voltage source, its electron detection time can be approximately calculated as:

$$
\left[\mathrm{C}_{\text {mod }} \times \mathrm{F}_{\text {mod }}\right]^{-1} \equiv \overline{\mathrm{t}} \equiv \frac{\overline{\mathrm{d}}}{\overline{\mathrm{E}} \times \mu_{\mathrm{e}}}=\frac{\mathrm{d}^{2}}{\mathrm{~V} \times \mu_{\mathrm{e}}}=\frac{15 \times 15 \mu \mathrm{m}^{2}}{1 \mathrm{~V} \times 1400 \mathrm{~cm}^{2} \mathrm{~V}^{-1} \mathrm{~s}^{-1}} \approx 1.6 \mathrm{~ns}
$$

The above calculation gives a reasonable estimation of the average photoelectron detection time for an electron generated not far from the surface if the internal potential along the photoelectron travel paths towards $n$ regions is monotonous and the electric field $(\mathrm{V} / \overline{\mathrm{d}})$ is constant along the photoelectron travel path. However, in practice, this assumption does not always hold true.

Firstly, in case of NIR light, the optical signal can penetrate deep into the substrate where the electric field is weaker. To overcome this effect, CAPD devices that are designed for NIR wavelengths can have an extra negatively biased bottom electrode to create a vertical electric field component [37]. Alternatively, the backside illumination may be used to confine the generated photoelectrons to a smaller volume [6]: the light, that passed through the photosensitive silicon layer, is reflected from the metallization layers and then reenters the photosensitive volume effectively increasing the photosensitive silicon thickness.

Secondly, when a modulation voltage source Vmix is connected to the biasing ohmic contacts ( $\mathrm{p}^{+}$regions) near the silicon surface, a slow drift region is formed under the ohmic contact with lower potential (illustrated with a dashed triangle in Figure 10) where the electric field is directed towards the substrate. Photo-electrons which are generated in this region are drifting towards a slow drift field region near the epi-substrate boundary and, when the polarity of the modulation voltage source Vmix is reversed, the photo-electrons are drifting towards the wrong detector $\mathrm{n}^{+}$-region lowering the demodulation performance. CAPD demodulation performance can be improved if the photogeneration under the ohmic contacts is prevented either by shielding, or by placing a microlens on top of the pixel focusing the light in the region with the strongest drift field ([6]), or by negatively biasing the substrate. Figure 12a-c [37] illustrates a CAPD architecture with a ring contact which is biased at a low potential and a modulation voltage Vmix is applied to the two ohmic contacts in the center. Such architecture reduces the mean travel path and the adverse effect of the slow region that may form under the negatively biased ohmic contact.

The assumption about monotonicity of the electrostatic potential may not hold in real devices leading to formation of charge pockets which deteriorate the device performance. Figure 12d illustrates the electrostatic potential under shallow trench isolation (STI). The potential around the $\mathrm{p}^{+}$-region, connected to the higher voltage, may have a local maximum due to the effect of the STI fixed positive charge. The Si/oxide interface has a positive surface-state charge which can vary in the order of $1 \ldots 10^{11} \mathrm{Q} / \mathrm{cm}^{2}$ depending on the technological conditions [38]. This positive charge bends energy bands forming charge pockets around the $\mathrm{p}^{+}$-region illustrated by the dashed red circle in Figure $12 \mathrm{~d}$. The charge pockets can trap photoelectrons leading to deterioration of the demodulation performance of CAPD sensor, as these trapped electrons can be detected by the wrong collector $\mathrm{n}^{+}$-region after the polarity of the modulation voltage source Vmix is reversed. The charge pockets around the $\mathrm{p}^{+}$-regions can be avoided by placing a negatively biased transparent electrode over the CAPD, as illustrated by Figure $12 \mathrm{~b}$, or by placing a donut shaped electrode around the $\mathrm{p}^{+}$-region where the charge pockets are formed [37], as illustrated by Figure 12c. The donut shaped electrode has an advantage over the solid electrode since reflection and absorption from polysilicon electrode does not lower the sensor responsivity as much, and the CAPD power consumption is not significantly increased because of formation of hole accumulation layer under the negatively biased electrode. Figure 12e illustrates the 
electrostatic potential under STI in the presence of the negatively biased electrode where the charge pocket was successfully removed.
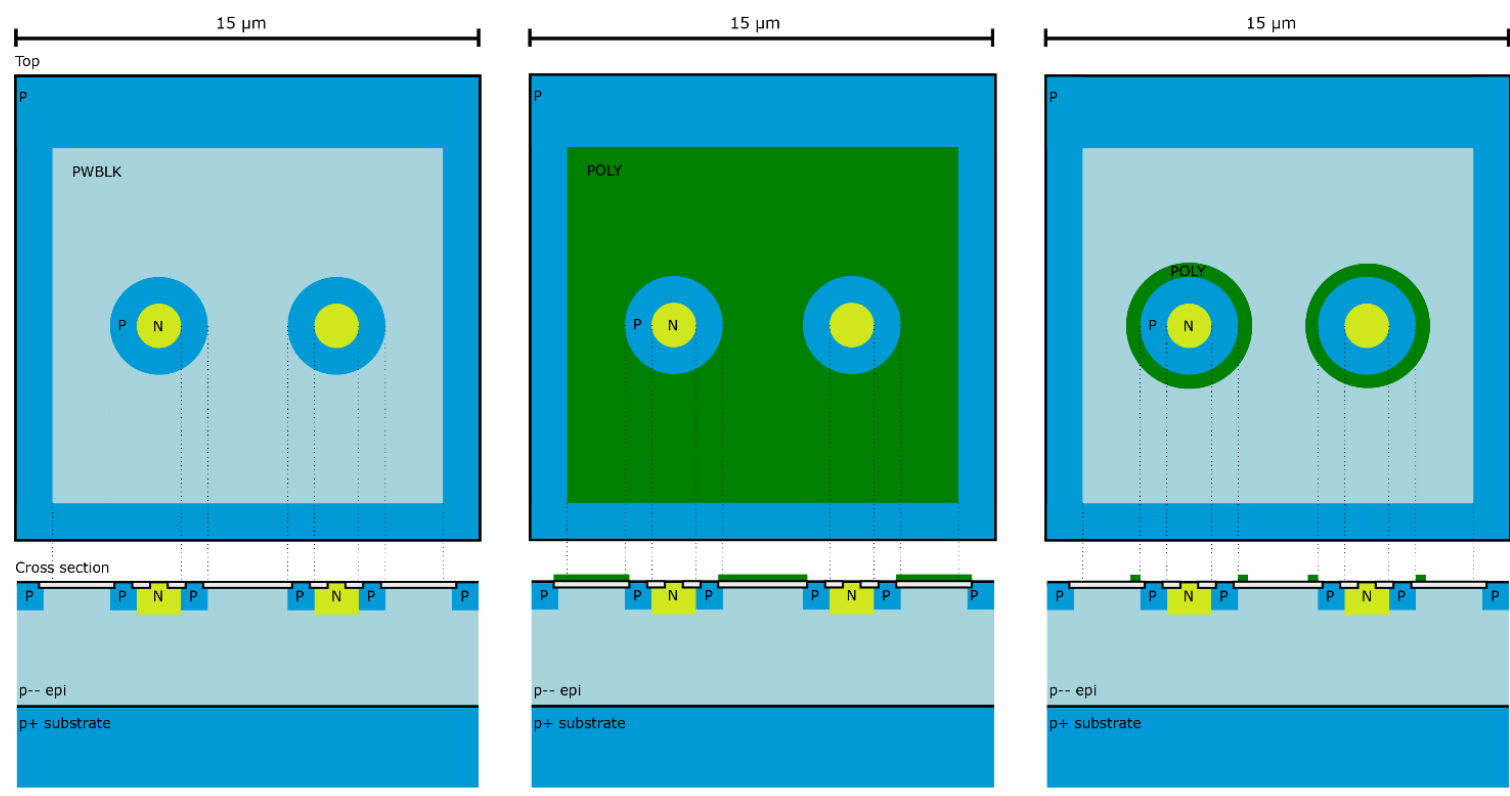

(a)

(b)

(c)

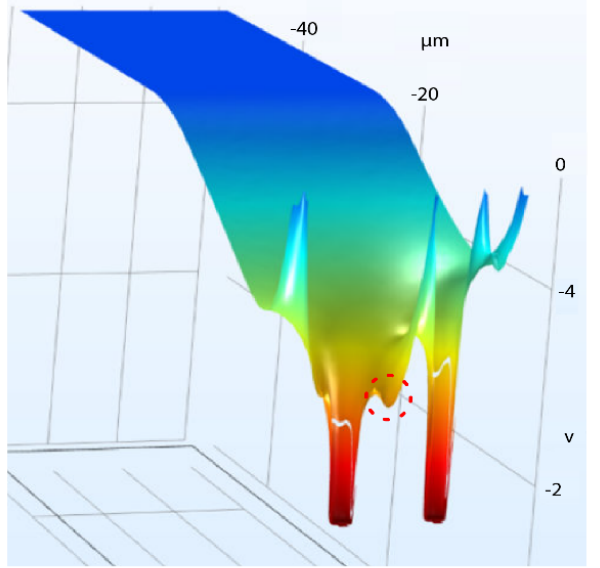

(d)

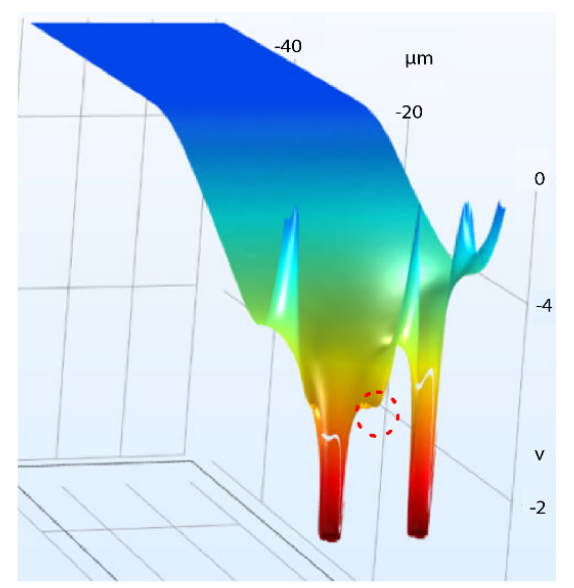

(e)

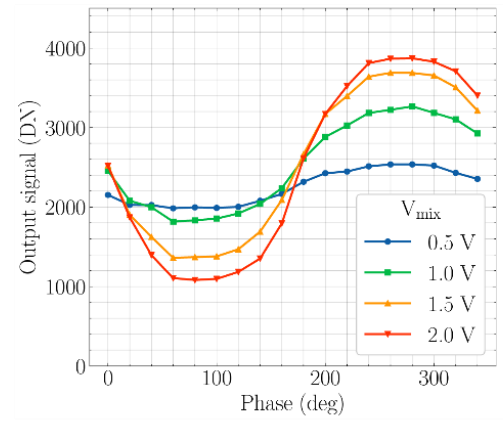

(f)

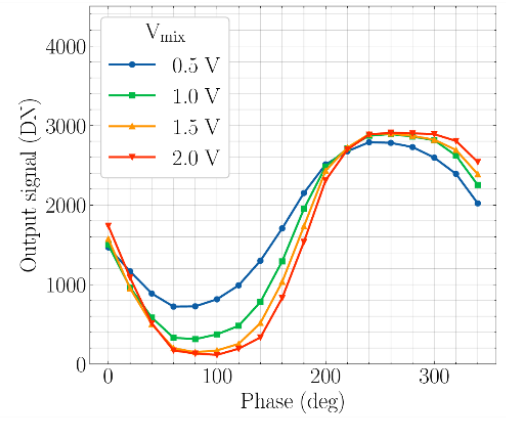

(g)

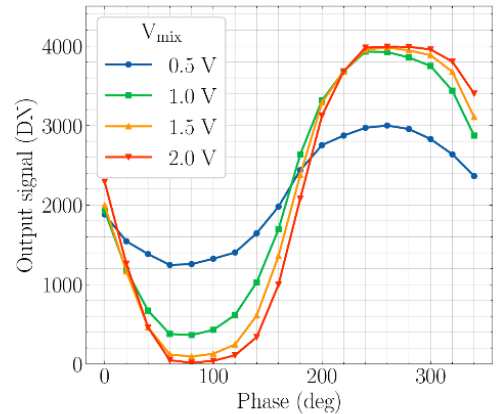

(h)

Figure 12. CAPD with a ring contact without a top polysilicon electrode (a), with a top polysilicon electrode (b), with a donut electrode $(\mathbf{c}, \mathbf{d})$ illustrates electrostatic potential under STI for device $(\mathbf{a}, \mathbf{e})$ illustrates electrostatic potential under STI for device $(\mathbf{b}, \mathbf{c})$ with a negative bias applied to the top electrode; $(\mathbf{f}-\mathbf{h})$ show measurement results of CAPD windowing function rect $\mathrm{CAPD}$ for $(\mathbf{a}-\mathbf{c})$ respectively. 
Figure $12 \mathrm{f}-\mathrm{h}$ show measurement results of CAPD windowing function rect ${ }_{\mathrm{CAPD}}$ measured by illuminating the sensor with a narrow $(\sim 3 \mathrm{~ns})$ optical pulse train signal and sweeping its phase while applying $50 \mathrm{MHz}$ square-wave signals to the $\mathrm{p}^{+}$-regions. The characterized CAPD devices have $15 \mu \mathrm{m}$ pitch and were manufactured in $180 \mathrm{~nm}$ technology. The CAPD without a transparent electrode over STI (f) shows poor demodulation performance due to charge pockets while having high signal amplitude (measured responsivity $\mathrm{R}=0.3 \mathrm{~A} / \mathrm{W}$ ). The CAPD with a transparent polysilicon electrode shows near ideal demodulation; however, the signal swing is reduced (measured responsivity $\mathrm{R}=0.2 \mathrm{~A} / \mathrm{W}$ ) compared to (f) due to reflection and absorption in the electrode. The CAPD device with the donut electrode $(\mathrm{h})$ combines good demodulation performance with higher responsivity (measured responsivity $\mathrm{R}=0.3 \mathrm{~A} / \mathrm{W}$ ).

\section{Conclusions}

The current-assistance principle allows to create strong electric drift fields in CAPD devices which are linearly distributed across large semiconductor volumes and which can penetrate deep in the semiconductor enabling high NIR sensitivity and cost-efficient manufacturing. CAPD devices are already widely used in numerous consumer and automotive applications.

Table 1 compares different CAPD implementations and the Kinect2 photonic demodulator.

Table 1. CAPD performance parameters.

\begin{tabular}{ccccc}
\hline Parameter & MLX75023 & $\begin{array}{c}\text { MLX75024 } \\
\text { (Donut Variant) }\end{array}$ & Kato et al. [39] & Kinect2 \\
\hline Tech. node & $0.35 \mu \mathrm{m}$ & $0.18 \mu \mathrm{m}$ & $90 \mathrm{~nm}$ & $0.13 \mu \mathrm{m}$ \\
Pixel pitch & $15 \mu \mathrm{m}$ & $15 \mu \mathrm{m}$ & $10 \mu \mathrm{m}$ & $10 \mu \mathrm{m}$ \\
$\mathrm{C}_{\text {mod } @ 50 \mathrm{MHz}}$ & $60 \%$ & $85 \%$ & $91 \%$ & $68 \%$ \\
R @ 850 nm & $0.2 \mathrm{~A} / \mathrm{W}$ & $0.3 \mathrm{~A} / \mathrm{W}$ & $0.34 \mathrm{~A} / \mathrm{W}$ & - \\
Pixel FF & $35 \%$ (native) & $70 \%$ (native) & $>80 \%$ ( $\mu$ lens) & $60 \%(\mu l e n s)$ \\
\hline
\end{tabular}

One of the further research frontiers for CAPD is extension of the spectral sensitivity range. The unique advantage of CAPDs over other common types of photonic demodulators is that the CAPD devices do not require high-quality oxides and can be manufactured in semiconductors like germanium which will allow sensing beyond the silicon sensitivity limit of $\sim 1100 \mathrm{~nm}$. Another potential research frontier is building CAPD sensors with a high number of taps that can be achieved, for example, by combining a gated demodulator with a current assistance principle [40].

\subsection{Current-Assisted Photonic Sampler (CAPS)}

The demodulation possibilities of current-assistance can also be optimized towards the creation of a sensor which operates as a fast shutter, i.e., in one state incoming light is detected with maximum efficiency and in a second state it is blind. Because photoelectrons generated in the second state do not need to be collected for readout but can just be disposed of, more compact and efficient structures can be employed for fast collection.

This way, a gated sensor was created and called the current-assisted photonic sampler as it samples the light in a certain gate time-window. Operation is equivalent to that of an ICCD, but without the gain. The CAPS was developed for fast-gated detection of NIR fluorescence lifetime in which sub-nanosecond decay rates of fluorescent molecules are imaged. The requirement for very fast time-gate edges was the incentive to create a current-assisted sensor optimized just for gating.

\subsubsection{Gated Detection of Fluorescence Lifetime}

The fluorescence lifetime $(\tau)$ is a parameter which describes the exponential decay rate of fluorescence after being excited with a pulse of light (Figure 13a). The fluorescence lifetime is the average time the molecule stays in an excited energy state after absorption 
of a photon and is an independent parameter which can be measured additionally to the spectral properties of fluorescence. The fluorescence lifetime depends on a number of things which each can be exploited for imaging:

1. The structure of the fluorescent molecule: imaging the lifetime can aid in revealing which fluorescent molecule is present or distinguish different fluorescent molecules [21].

2. The chemical environment of the fluorescent molecule: fluorescence lifetime has a sensitivity to chemical conditions such as $\mathrm{pH}$ or $\mathrm{Ca}+$ or $\mathrm{O}_{2}$ concentrations and can as such be used as a probe for these conditions [41].

3. The distance to other fluorescent molecules: the fluorescence lifetime of a fluorescent molecule is influenced by another fluorescent molecule whose absorption spectrum overlaps with the emission spectrum of the first molecule and the influence is dependent on the proximity of the molecules. This property is exploited in a technique called time-domain FRET which is one of the most popular applications of fluorescencelifetime imaging because the fluorescence-lifetime offers an absolute measure for the proximity in contrast to the relative intensities used in classic FRET [42].

4. The mobility of the fluorescent molecule: a molecule which is mechanically bound will lose less energy through non-radiative processes [43].
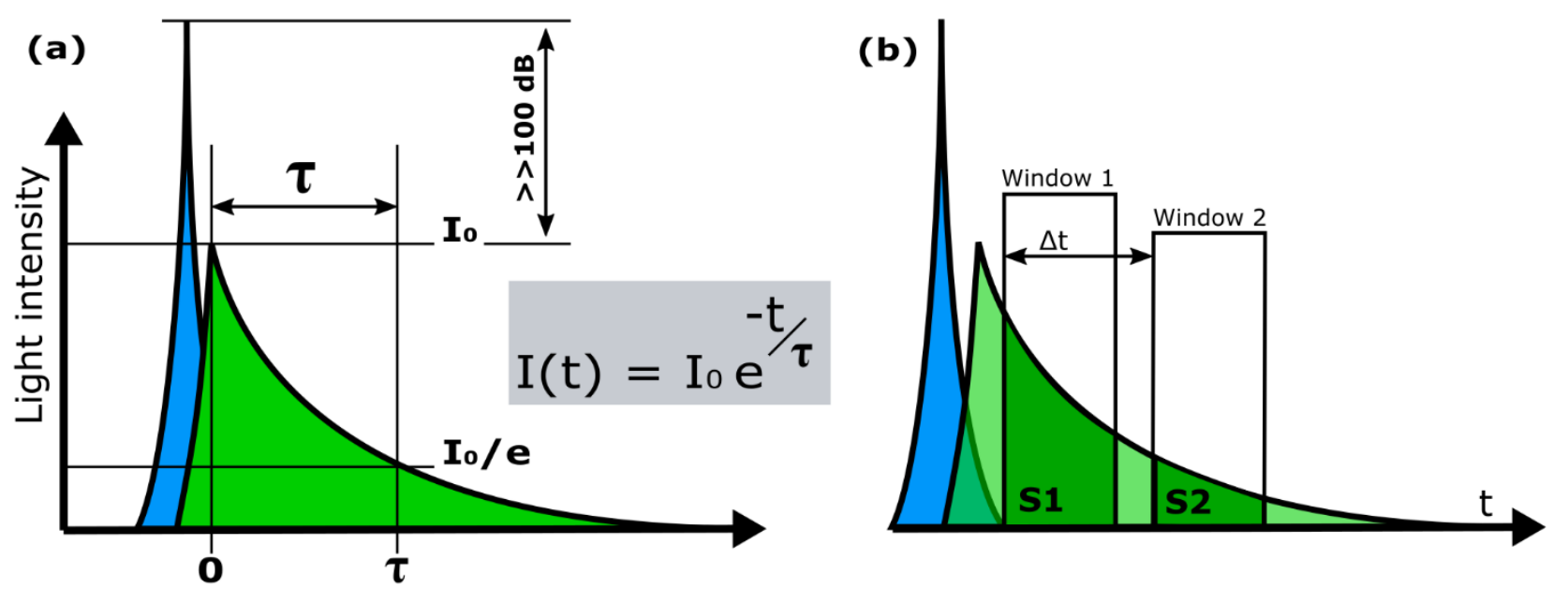

Frame 1

(c)
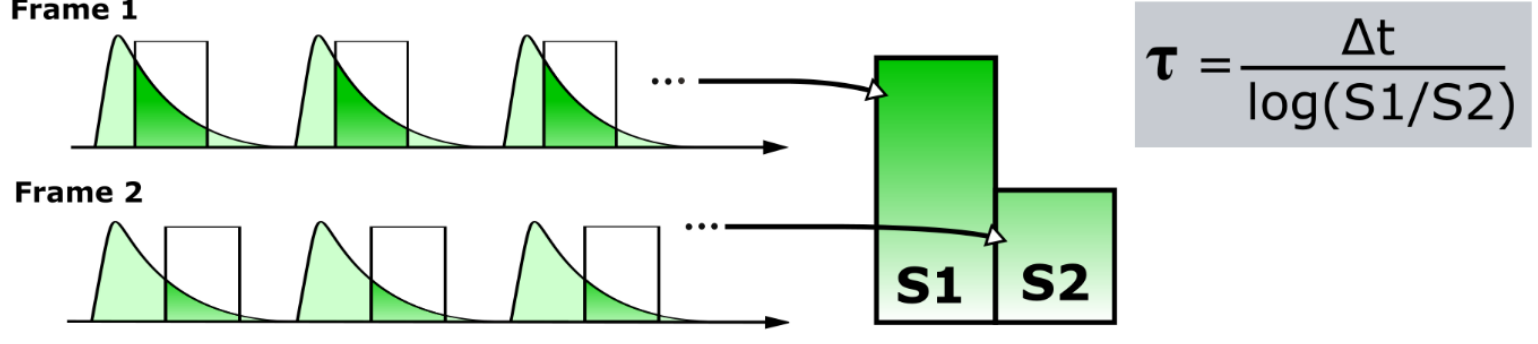

Figure 13. Fluorescence lifetime $(\tau)$ definition (a) 2-window gated lifetime measurement (b) and (practical implementation of the 2-window method for a gated camera (c).

The fluorescence lifetime is typically in the nanosecond range and lifetimes as short as several hundreds of picoseconds are not an exception for NIR fluorescent molecules. This poses severe challenges for any system measuring and even imaging lifetimes. The gold standard in fluorescence-lifetime measurements is time-correlated single-photon counting (TCSPC) in which the arrival time of single photons is recorded and stored in a histogram which can then be analyzed with computationally expensive fitting methods. Implementing the electronics required for TCSPC in every pixel of an image sensor is difficult and TCSPC is usually either performed with a discrete single-photon detector for the analysis of a substance or with a scanning system for imaging in microscopy. 
Fluorescence lifetime can also be detected with a gated detector in a family of methods which is called Rapid-Lifetime Determination (RLD). Figure 13b demonstrates the simplest incarnation of RLD in which the fluorescence intensity is measured in two equal timewindows at two different positions in the fluorescent decay separated by a time $\Delta \mathrm{t}$. In the ideal case of a mono-exponential decay without background, the fluorescence lifetime can simply be calculated from $\Delta t$ and the ratio of the signal detected in the two windows. The method can be extended to three windows to account for background signal and optimized window spacings and widths can be used for optimized lifetime measurement precision. Imaging fluorescence lifetime with an image sensor which implements a single gate-window can be performed by taking two frames at two different gate-window time positions. In each frame signal is collected from many consecutive fluorescence excitations. Finally, the lifetime is calculated from the integrated signal from frame 1 and frame 2 (Figure 13c).

\subsubsection{CAPS Operation Principle}

The CAPS sensor has some features in common with the CAPD: the sensor is placed in a lowly-doped $p^{-}$-substrate and around the sensor is a ring substrate contact at a negative potential, creating a substrate drift field that accelerates photo-generated electrons towards the center of the sensor.

The CAPS sensor consists of a centrally placed detection node surrounded with four drain nodes as shown in Figure 14a. The detection node consists of a $\mathrm{n}^{+}$cathode surrounded by a $\mathrm{p}^{+}$anode. Since the drain node is used to evacuate the carriers, a more compact structure is used, where $\mathrm{n}^{+}$and $\mathrm{p}^{+}$regions are abutted. Because the silicide layer provides conduction between the $\mathrm{n}^{+}$and $\mathrm{p}^{+}$region, a single contact can both drain away photo-generated electrons and apply the necessary hole currents for the generation of the drift field. As such, the $\mathrm{p}^{+}$zone can be made narrower, resulting in a very short diffusion distance.

By setting the drain nodes to a higher potential than the detection node, the detection node becomes completely shielded from photo-generated electrons. In this situation the gate is said to be OFF. If instead the detection node is set to the highest potential, electrons will be allowed to flow into the detection node and the gate is turned ON [20].

Figure $14 b, c$ show the gating effect in more detail. On the left, the complete shielding of the detection node can be seen when the gate is OFF. Electrons travel towards higher potentials and as such electrons from anywhere in the detector will end up at the drain nodes and not at the detection node. This results in very good gate OFF quality or high gating contrast (difference in detected signal between detector in $\mathrm{ON}$ and detector in OFF state). When the gate is $\mathrm{ON}$, following the highest potential leads directly to the detection node and leads to high-speed detection of electrons.

This fast gating mechanism does not require the use of an image intensifier, avoiding the photocathode efficiency loss found in ICCD cameras. Furthermore, this gating mechanism requires modulation of only low voltages which can be handled by high-speed electronics at high repetition rates.

Figure 15 shows the typical potentials involved in the CAPS operation. The detection node is connected to a 3T-readout circuit for resetting of the detection node and buffering of the sense-node voltage by a source follower. At the start of every measurement, the sense node is reset by asserting the RST-signal. After having reset the sensor, the gate signal will be asserted at a fixed position in time with respect to the measured light and this for $n$ cycles. In practice, this usually means that the gate is synchronized to the pulsed laser that excites the fluorescence and that the emission is gated for $\mathrm{n}$ repetitions of the laser pulse, during which the signal is integrated. The voltage on the sense node is then sampled by an $\mathrm{ADC}$ and stored as measurement result for the gate in position 1 . After this first integration period, a new integration period starts with the gate in another position and the resulting voltage is sampled again. These two results can then be used to estimated fluorescence 
lifetime as described in Section 3.3.1 or the integration process can be repeated with the gate in more positions for extra measurement points.

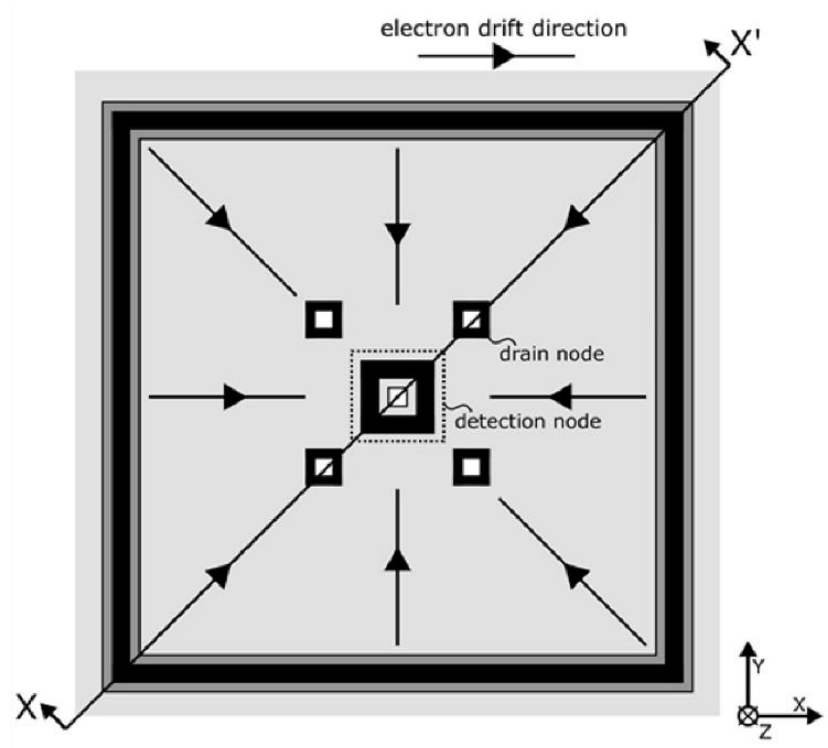

(a) CAPS top view
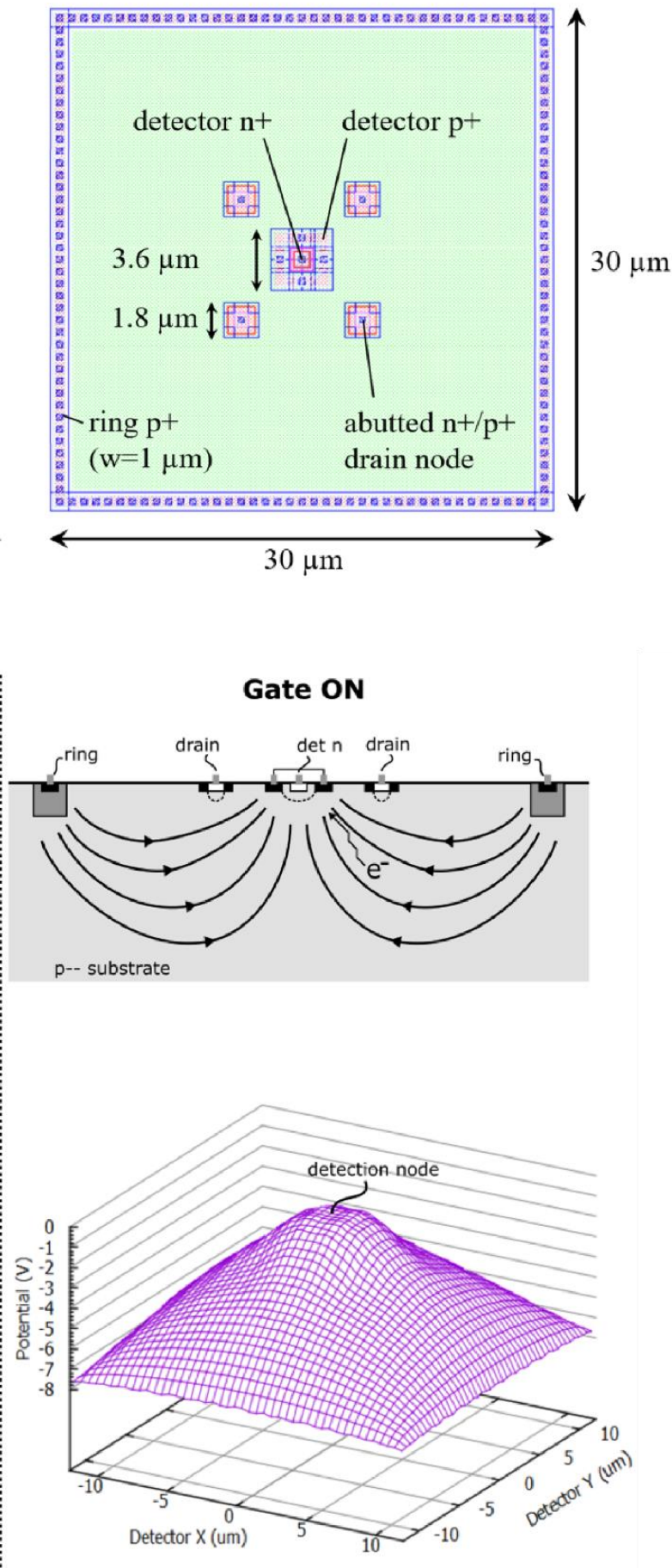

(c) Potential at $Z=1.3 \mu \mathrm{m}$

Figure 14. CAPS principle showing a top view of a CAPS sensor with detection node and four drain nodes with indication of drift field (a, left) and top view of the layout with dimensions indicated (a, right). CAPS cross section when the gate is $\mathrm{OFF}$ and when the gate is $\mathrm{ON}(\mathbf{b})$ and associated potential distributions at a depth of $1.3 \mu \mathrm{m}(\mathbf{c})$. 


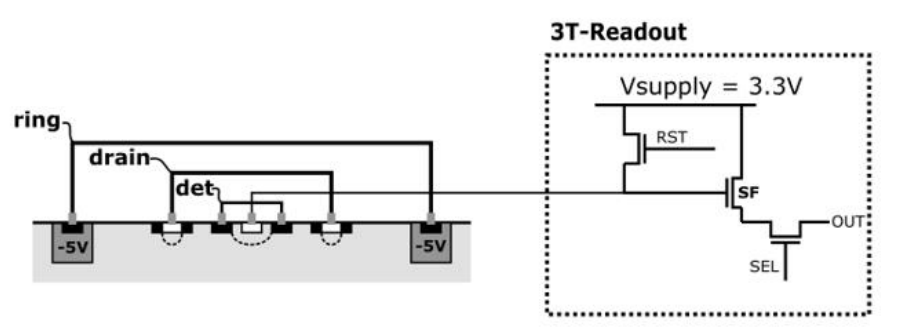

(a) Sensor with substrate contacts and readout circuit

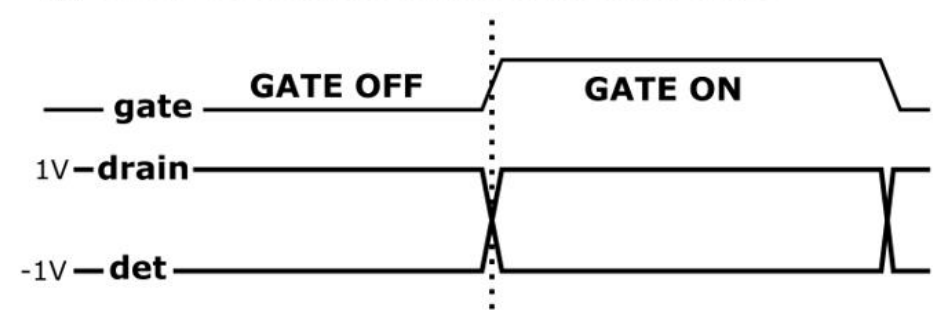

(b) Gate control signals

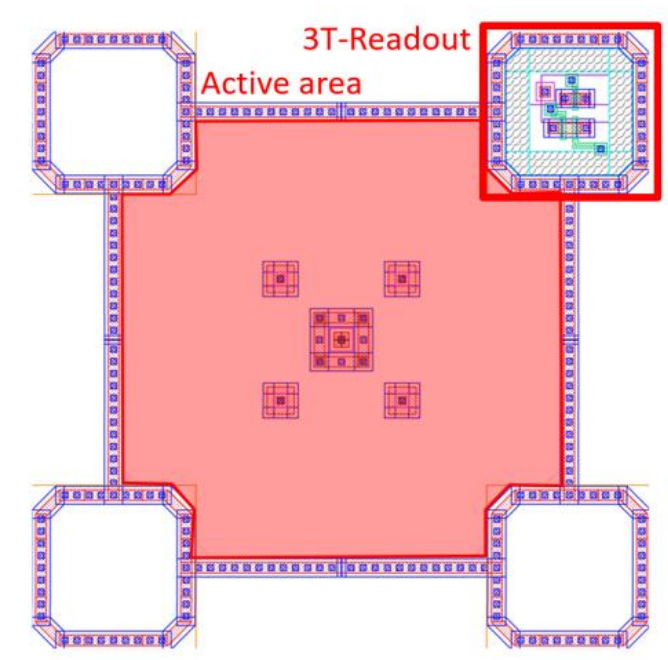

(c) Layout with readout circuitry

Figure 15. Typical operation of a CAPS sensor showing the sensor substrate contacts and 3T readout circuit (a) and gating control signals $(\mathbf{b})$. The layout of the CAPS sensor with readout circuitry is shown in (c).

\subsubsection{CAPS Sensor Characteristics}

Unlike the CAPD, the CAPS is not a symmetrical device. Due to its design with a centrally placed detection node surrounded by drains, it will be easier to direct electrons into the drains than into the detection node. This can be seen in the DC gating behavior, shown in Figure 16a: with increasing positive differential gating voltages, the photocurrent into the detection node rises more slowly than it decreases for negative voltages. The reason for the slower increase in detected electrons at positive gating voltages is the fact that electrons have to be directed past the drain nodes. It can also be seen that the ring voltage should not be too negative to achieve a high gating contrast and a sharp curve. The latter is important when the pixel is placed in an array: a voltage drop over the gating signal lines should not result in a different gating behavior.

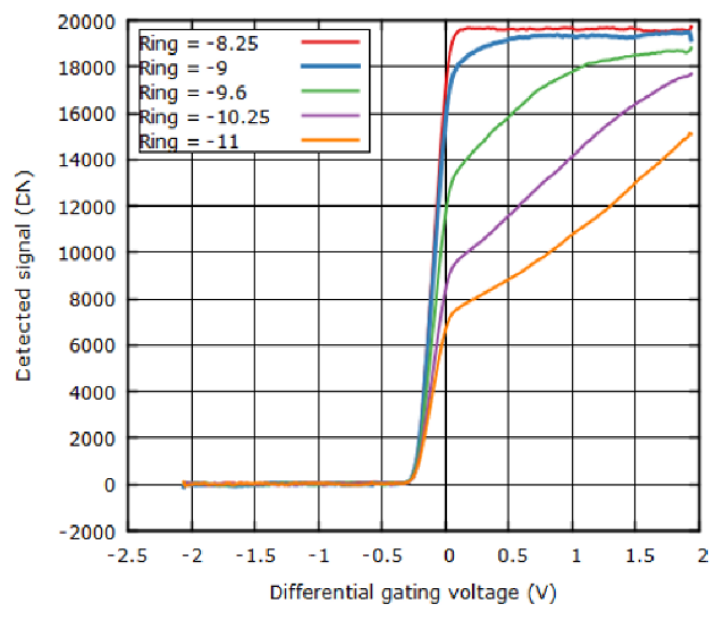

(a)

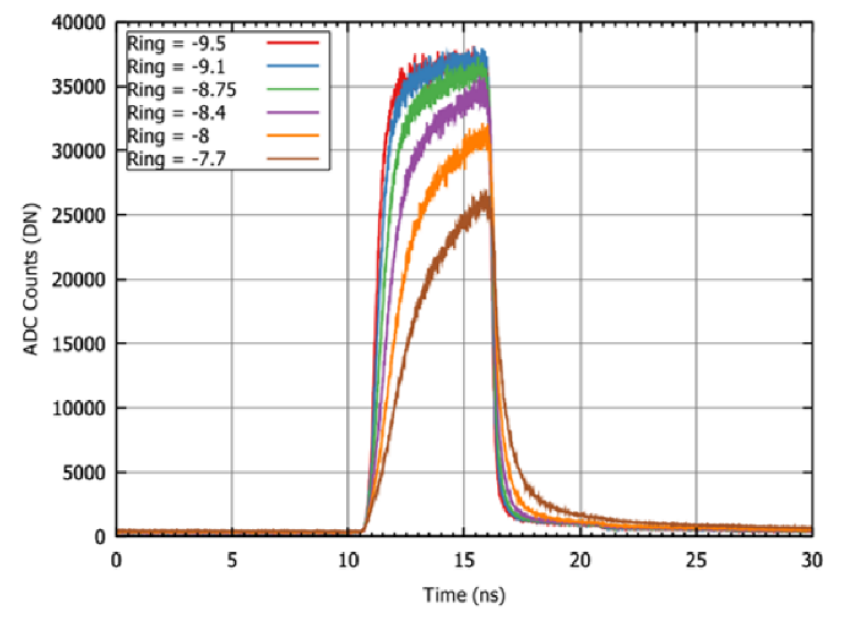

(b)

Figure 16. Gating behavior of the CAPS sensor in DC (a) and instrument response function (IRF) for a 4 ns gate (b).

Figure $16 \mathrm{~b}$ shows a plot of the Instrument Response Function (IRF) for a 4 ns gating width at multiple ring voltages. This measurement allows to directly assess the lifetime measurement capabilities: the slope of the falling edge is used to determine the intrinsic decay time, while the rising edge determines the minimum gate window. It can be seen that at less negative ring voltages, the IRFs decay rate becomes slower, but also the IRF 
rises slower and at some point does not reach full amplitude. Therefore, more negative ring voltages are used for a higher $\mathrm{QE}$ and short gate widths. In practice, an optimum voltage is found where both a high gating contrast and high speed are achieved.

\subsubsection{Fluorescence Lifetime Imaging}

A first-generation $32 \times 32$-pixel CAPS array has been realized in a standard $350 \mathrm{~nm}$ CMOS process on a high resistivity $(\sim 1000 \Omega \cdot \mathrm{cm})$, thick p-type epilayer $(14 \mu \mathrm{m})$. A camera is developed around this to demonstrate the capabilities of the CAPS-array [21]. The pixels in the array measure $30 \times 30 \mu \mathrm{m}$ with an active area of $480 \mu \mathrm{m}^{2}$ resulting in a fill factor of $53 \%$. The camera characteristics, listed in Table 2, are results for pixel $[16,16]$ located in the center of the array. These characteristics are compared to commercially available Intensified CCD cameras in Table 3. A micrograph of the sensor die and the camera setup can be seen in Figure 17.

Table 2. Proof-of-concept CAPS camera characteristics.

\begin{tabular}{cc}
\hline Resolution & $32 \times 32$ \\
\hline Pixel size & $30 \times 30 \mu \mathrm{m}$ \\
Fill factor & $53 \%$ \\
@780 nm & $25 \%$ \\
External (effective) quantum efficiency & $71 \%$ \\
Internal (effective) quantum efficiency & $500 \mathrm{ps}$ \\
Minimum gate width & Up to the laser repetition period \\
Maximum gate width & 11 ps \\
Gate position time resolution & Full laser repetition range \\
Gate position range & $>100 \mathrm{MHz}$ \\
Maximum gate repetition rate &
\end{tabular}

Table 3. CAPS proof-of-concept camera compared to commercially available Intensified CCD cameras.

\begin{tabular}{ccccc}
\hline Camera & Photocathode & $\begin{array}{c}\text { NIR QE } \\
\text { @ 780 nm }\end{array}$ & $\begin{array}{c}\text { Minimum Gate } \\
\text { Window }\end{array}$ & $\begin{array}{c}\text { Maximum Gate } \\
\text { Repetition Rate }\end{array}$ \\
\hline La Vision PicoStar HR [44] & Gen II & $<8 \%$ & $300 \mathrm{ps}$ & $110 \mathrm{MHz}$ \\
Andor iStar U [45] & Gen II & $<10 \%$ & $2 \mathrm{~ns}$ & $500 \mathrm{kHz}$ \\
Andor iStar U [45] & Gen III & $<24 \%$ & $2 \mathrm{~ns}$ & $500 \mathrm{kHz}$ \\
Princeton Instruments PI-MAX4 [46] & Gen II & $<12 \%$ & $500 \mathrm{ps}$ & $100 \mathrm{kHz}$ \\
Princeton Instruments PI-MAX4 [46] & Gen III & $<27 \%$ & $500 \mathrm{ps}$ & $100 \mathrm{kHz}$ \\
CAPS camera today & NA & $<25 \%$ & $500 \mathrm{ps}$ & $>100 \mathrm{MHz}$ \\
CAPS camera possibility & NA & $<71 \%$ & $500 \mathrm{ps}$ & $>100 \mathrm{MHz}$ \\
\hline
\end{tabular}

Practical use of this $32 \times 32$ CAPS image sensor has been demonstrated in a proof-ofconcept for fluorescence-guided surgery [18]. Fluorescence contrast is increasingly used in surgical guidance to light up blood flow, tumors, nerves, and more. Because of its ability to penetrate a few millimeters of tissue, the wavelength of preference is around $800 \mathrm{~nm}$. This leaves little spectral room to image more than one contrast agent based on spectral differences. In the proof-of-concept, the CAPS image sensor is used for fluorescence-lifetime imaging of an ex-vivo mouse phantom with two $800 \mathrm{~nm}$ fluorescence inclusions of dyes (ICG and IRDye-800CW) but with a different fluorescence lifetime [18]. The CAPS camera is able to distinguish both dyes based on a difference in fluorescence lifetime whereas a conventional fluorescence camera cannot. More recently, at the EMIM 2020 conference on molecular imaging, the same camera has been demonstrated in an in vivo experiment. In the experiment, a tumor-bearing mouse was injected with an anti-EGFR nanobody-based fluorescence contrast agent. When imaged in conventional fluorescence mode the tumor lights up. However also the kidneys light up. Only in the CAPS fluorescence-lifetime image can the difference between kidneys and tumor be seen, demonstrating that fluorescence- 
lifetime can be used as modality to distinguish Fluorescence emission from the targeted tissue (tumor) and non-specific emission (in this case kidneys) (Figure 18).

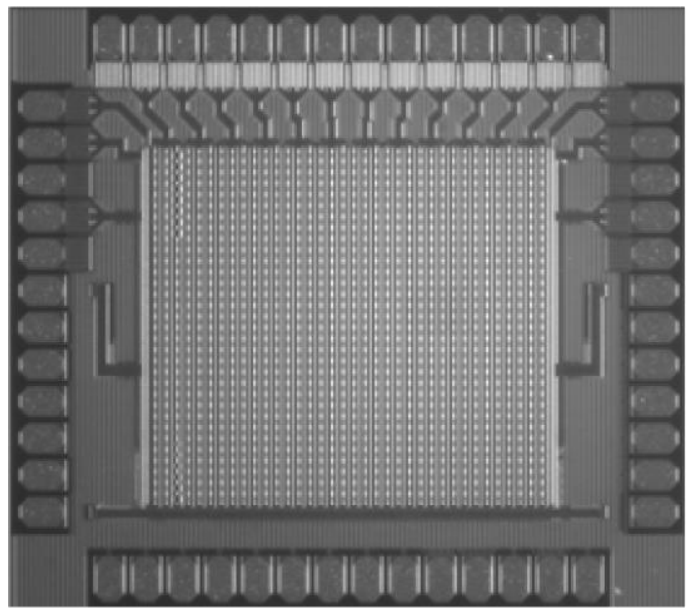

(a)

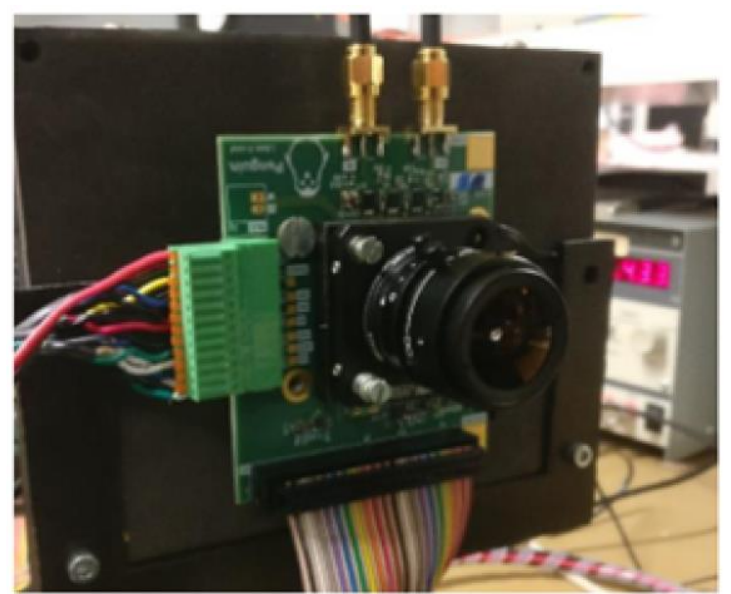

(b)

Figure 17. Micrograph of the $32 \times 32$-pixel CAPS array (a) and camera setup (b).

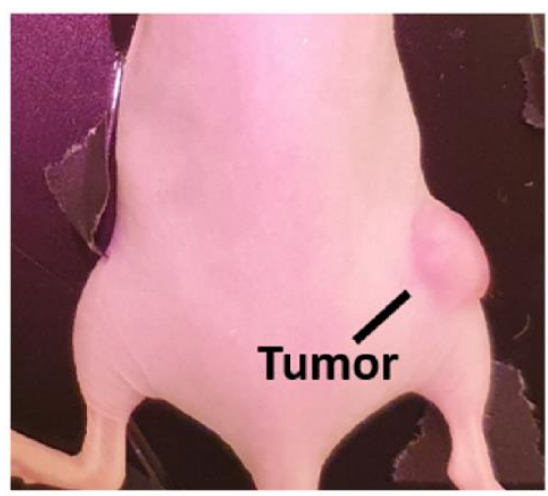

(a)

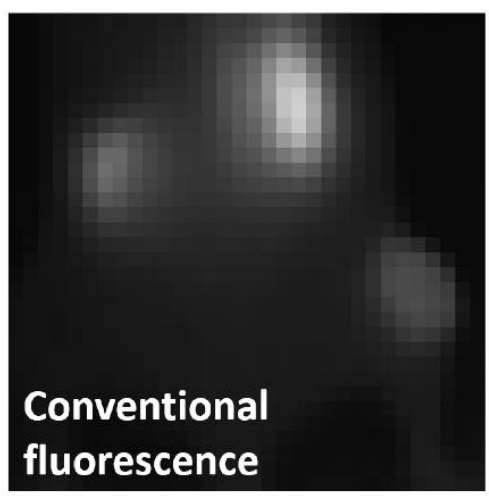

(b)

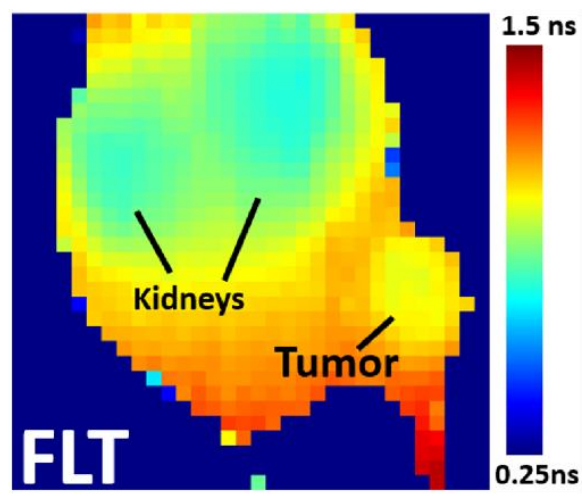

(c)

Figure 18. In vivo fluorescence experiment using a CAPS fluorescence-lifetime camera. A mouse bearing a xenograft tumor with EGFR expression and injected with an anti-EGFR nanobody-based NIR fluorescence contrast agent (a). Both tumor and kidneys light up under conventional fluorescence imaging (b). CAPS fluorescence-lifetime imaging reveals a fluorescence lifetime difference between the tumor and kidneys (EMIM 2020) (c).

\subsubsection{Conclusions}

By combining good NIR quantum efficiency with fast gating, the CAPS image sensor seems a good candidate for in vivo fluorescence imaging in which NIR dyes play a crucial role and in which a fluorescence-lifetime imaging capability can enable new possibilities for future applications such as the imaging of fluorescence contrast agents simultaneously in complex surgical procedures or increasing the contrast specificity by taking fluorescence lifetime contrast into account.

\subsection{Current-Assisted SPAD (CA-SPAD)}

Single-photon avalanche diodes (SPADs) are widely popular due to their singlephoton sensitivity and CMOS compatibility [47-53]. CMOS compatibility and inherent digital nature of the SPAD detection leads to quenching and timing processing circuitry to be constructed around the SPAD pixel. A recent work presenting the realization of a Megapixel SPAD imager further demonstrates the advancements made in the field of 
SPADs fabricated in CMOS technologies [47]. Frontside-illuminated (FSI) SPADs have limited NIR sensitivity, often because the device is enclosed in a deep N-well, in order to isolate the moderately high voltages of the SPAD. As seen in Section 2, increasing the absorption layer thickness alone does not lead to an increase in photo-sensitivity. Therefore, an idea of a novel SPAD detector with a small p-n junction and a large photo-absorption volume with drift field was conceived. The drift field is due to the potential gradient in the absorption volume, arising from applying a bias between two terminals. In short, we integrate the current-assistance principle with a small junction SPAD to increase its photo-absorption volume with swift response speed. To stick with the convention, this novel SPAD is called "current-assisted SPAD (CA-SPAD)".

The first proof-of-concept device (we will refer to it as CA-SPAD-1 [24]) was fabricated in X-fab foundry's $350 \mathrm{~nm}$ CMOS (XO035). Mask layers readily available for standard transistor formation were used and no special layers were used. Hence, the fabrication is done in a relatively low-cost and accessible CMOS technology. This technology has a high resistivity $(\sim 1000 \Omega \cdot \mathrm{cm})$, thick p-type epilayer $(14 \mu \mathrm{m})$ which enables possibility of good NIR sensitivity. The illustrated cross-section is shown in Figure 19a. The central SPAD is comprised of a $\mathrm{N}$-well cathode surrounded by a $\mathrm{p}^{+}$anode. $\mathrm{N}$-well is chosen covering the $\mathrm{n}^{+}$doping, to form the cathode (detect node in other current-assisted detectors), to reduce band-to-band tunneling at large reverse bias voltages. A consequence of having an $\mathrm{N}$-well is the increased breakdown voltage. This central p-n junction is surrounded by a much larger $\mathrm{p}^{+}$"ring", which marks the boundary of the photo-detection area. A potential difference is applied between the anode and the ring, which results in drift field between them. The anode is biased more positively with respect to the ring in order to attract the photo-generated electrons towards the anode. The photoelectrons which reach the anode will have to only diffuse a small distance before falling into the depletion region and commencing an avalanche breakdown. There is a steady hole current between the anode and the ring, due to the potential difference, leading to a steady power dissipation per pixel which is a small price to pay to enable a high-speed operation.

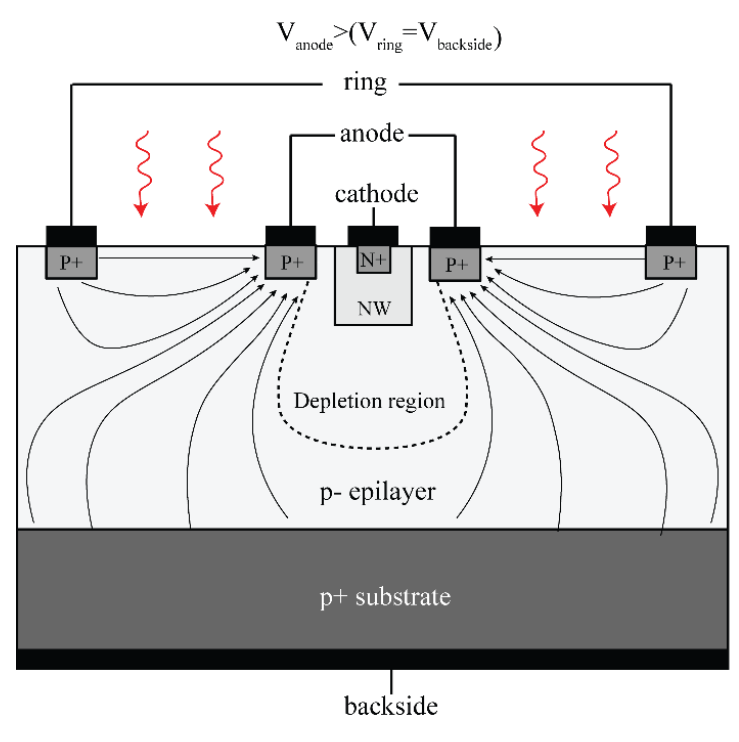

(a)

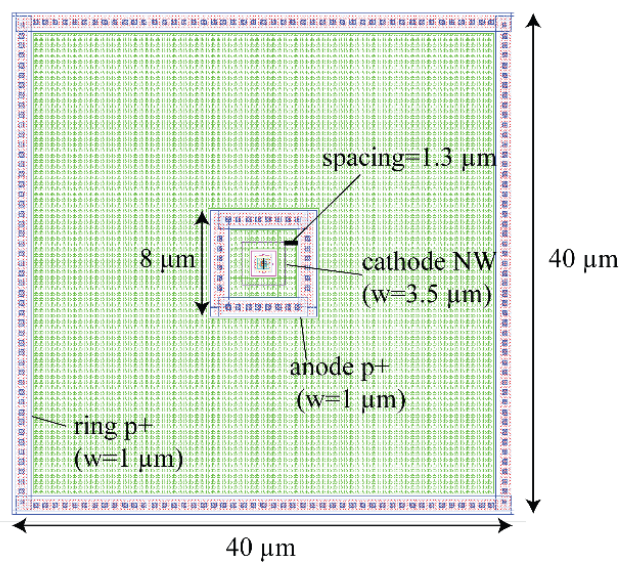

(b)

Figure 19. Cross section of CA-SPAD-1, lines with arrows indicate the direction of photo-electrons, dotted line represents the boundary of the depletion region (a) and top-view of the layout of CA-SPAD-1 with dimensions indicated (b).

CA-SPAD-1 had square structures which are spaced adequately (shown in Figure 19b) to comply with the design rules with some margin. Passive quenching (external to the chip) was used to characterize the device behavior and a few important issues were identified. The primary issue was the sharp corners created by the square geometry. Sharp corners 
are known to concentrate the electric field which lead to selective breakdown [54-56]. This resulted in limited photon detection probability (PDP) and increased timing jitter due to the non-uniform electric field. Light emission tests show discrete light emission spots from sharp corners, further suggesting that the breakdown occurs primarily at the sharp corners (shown in Figure 20). The secondary issue was the lack of on-chip quenching circuitry which resulted in large deadtimes ( $50 \mathrm{~ns})$ due to increased parasitic capacitance from the bondpads $(\sim 1 \mathrm{pF})$. While the SPAD p-n junction capacitance is estimated to be $\sim 1 \mathrm{fF}$, large parasitic capacitance also means that there is more current required to quench the avalanche operation. This results in more carriers flowing through the p-n junction during an avalanche event which increases the after pulsing probability (APP). Although the device did not perform up to the existing FSI SPADs, it was vital in demonstrating that the principle works as expected and the main issues were identified.

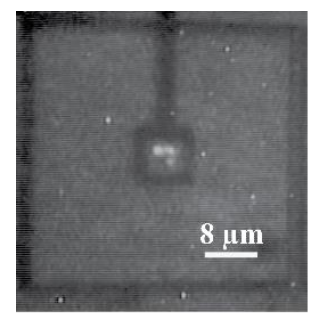

$$
\mathrm{V}_{\mathrm{cx}}=2.1 \mathrm{~V}
$$

(a)

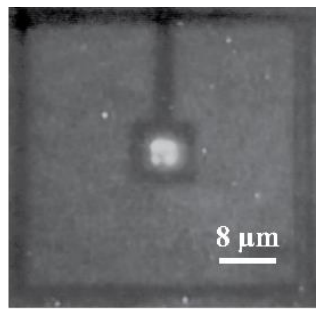

$\mathrm{V}_{\mathrm{cx}}=2.8 \mathrm{~V}$

(b)

Figure 20. Light emission test to demonstrate reverse breakdown occurring at sharp corners at $\mathrm{V}_{\mathrm{ex}}=2.1 \mathrm{~V}(\mathbf{a})$ and $\mathrm{V}_{\mathrm{ex}}=2.8 \mathrm{~V}(\mathbf{b})$.

The second iteration (we will refer to it as CA-SPAD-2 [25]) was also fabricated in X-Fab's XO035 technology. A few changes were made in the design of the pixel: (1) The junction was made with a cylindrical symmetry to avoid sharp corners (shown in Figure 21), (2) The "ring" terminal is chosen to be a P-well (with a $\mathrm{p}^{+}$contact) to realize a more ohmic contact, (3) The pixel pitch was reduced to $30 \times 30 \mu \mathrm{m}$. An on-chip passive quenching resistor $(100 \mathrm{k} \Omega)$ was used for quenching.

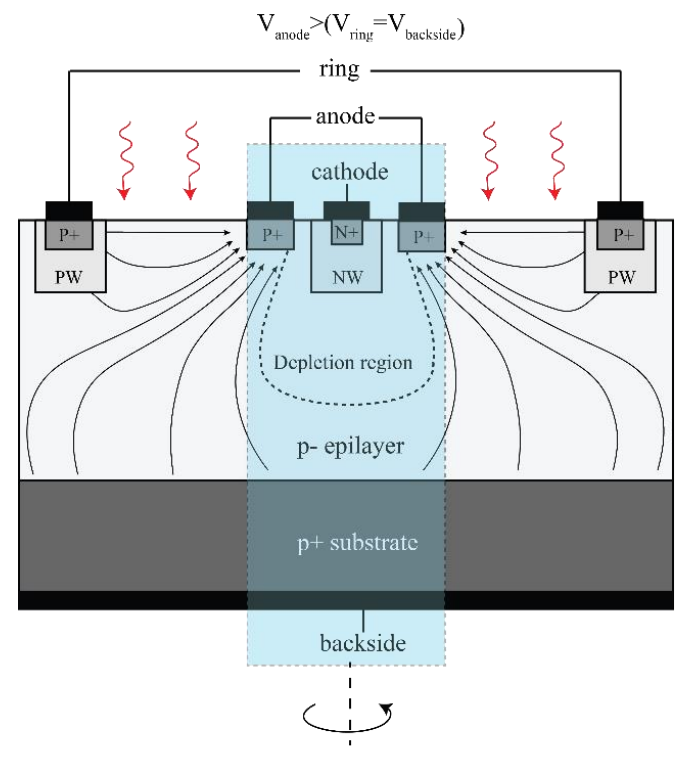

(a)

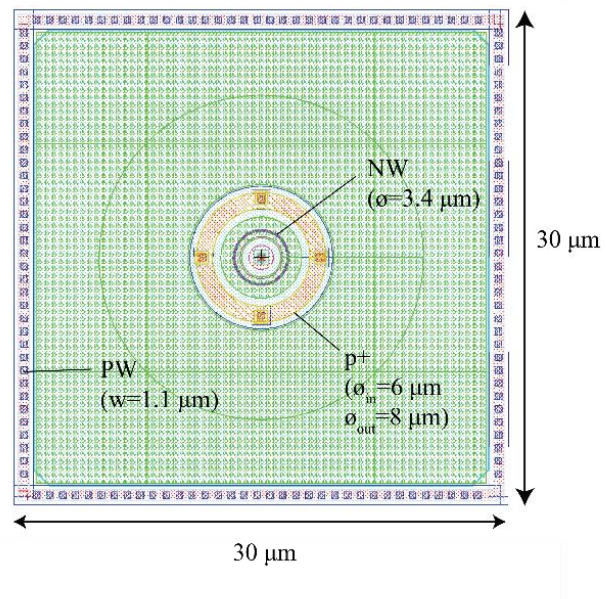

(b)

Figure 21. Cross section of CA-SPAD-2, lines with arrows indicate the direction of photo-electrons, the dotted line represents the boundary of the depletion region (a) and top-view of the layout of CA-SPAD-2 with dimensions indicated (b). 
A light-emission test for CA-SPAD-2, shown in Figure 22, shows one uniform light emission spot suggesting that breakdown is uniform in the p-n junction. This is also confirmed by the improved timing precision shown in Figure 23b and photo detection probability shown in Figure 23d. Dark count rate is still large, shown in Figure 23a and is primarily due to the $\mathrm{N}$-well $/ \mathrm{p}^{+}$junction construction. Afterpulsing probability at $\mathrm{V}_{\mathrm{ex}}$ of $2.5 \mathrm{~V}$ is $13 \%$ which could possibly be reduced by efficient quenching circuits.

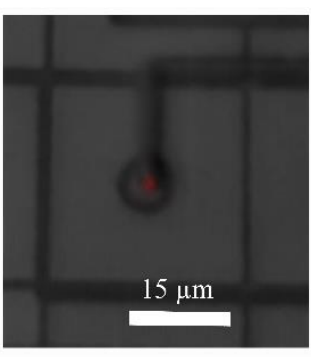

$$
\mathrm{V}_{\mathrm{ex}}=1.5 \mathrm{~V}
$$

(a)

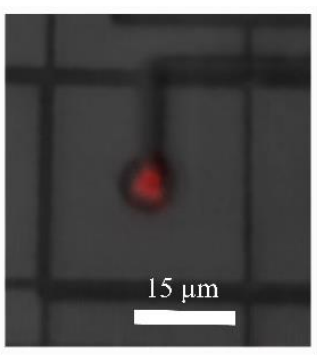

$\mathrm{V}_{\mathrm{ex}}=2 \mathrm{~V}$

(b)

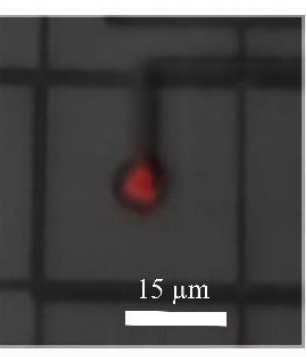

$\mathrm{V}_{\mathrm{ex}}=2.5 \mathrm{~V}$

(c)

Figure 22. Light emission from "central SPAD" area at $\mathrm{V}_{\mathrm{ex}}=1.5 \mathrm{~V}(\mathbf{a}), \mathrm{V}_{\mathrm{ex}}=2 \mathrm{~V}(\mathbf{b})$ and $\mathrm{V}_{\mathrm{ex}}=2.5 \mathrm{~V}$ (c). The light emission is false-colored red.

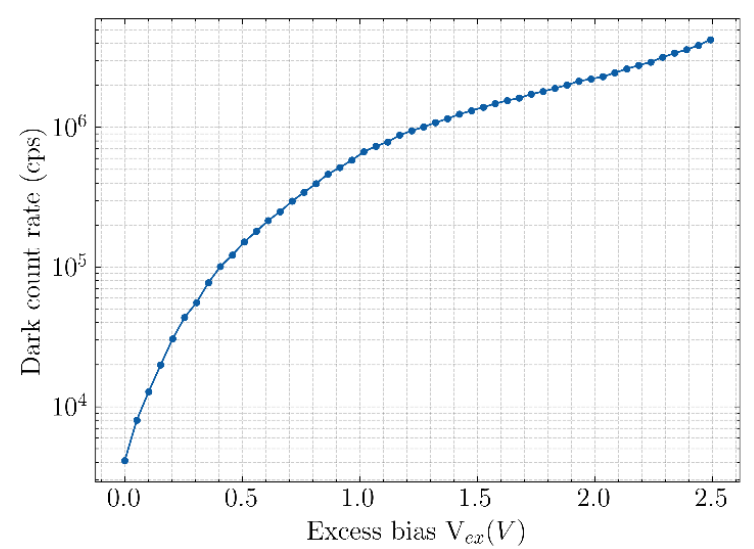

(a)

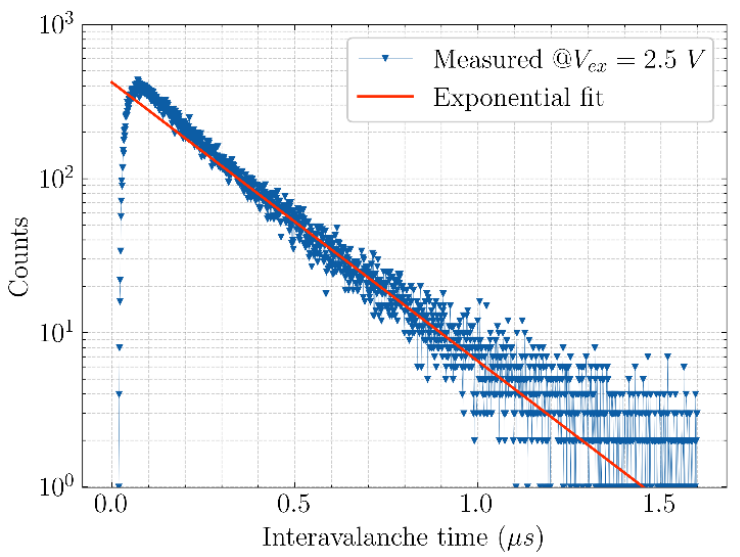

(c)

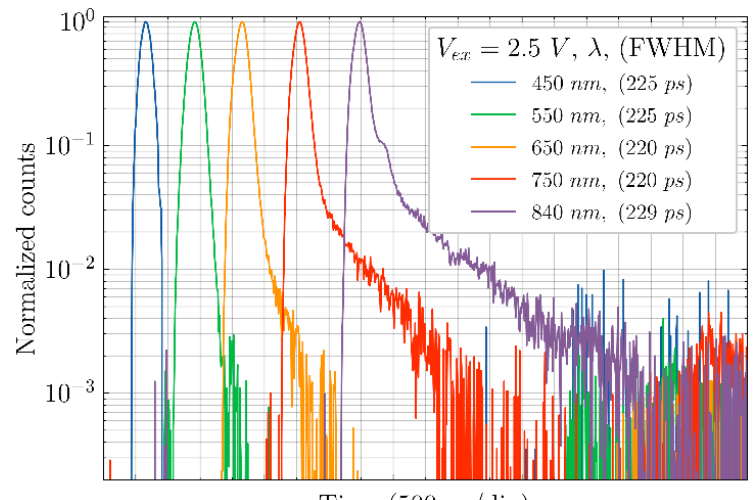

Time $(500 \mathrm{ps} / \mathrm{div})$

(b)

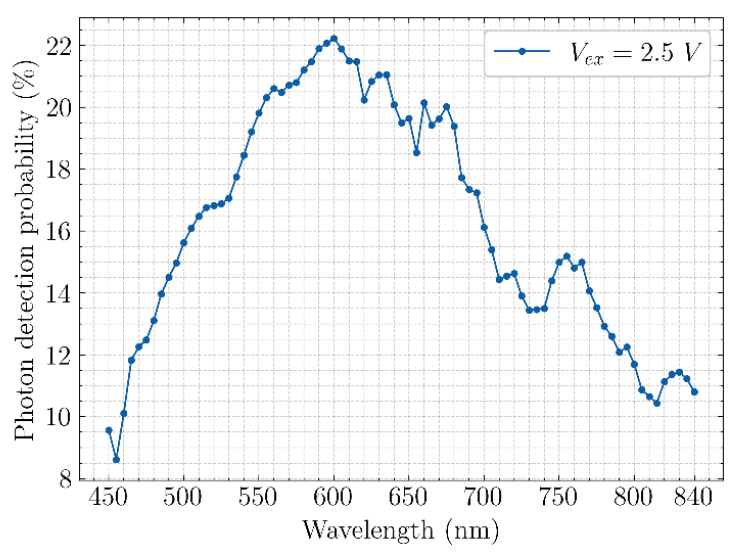

(d)

Figure 23. Dark count rate as a function of excess bias voltage for CA-SPAD-2 (a), Timing response for different wavelengths for CA-SPAD-2 (b), Inter-avalanche histogram to characterize after-pulsing probability for CA-SPAD-2 (c), and photon detection probability (PDP) as a function of wavelength for CA-SPAD-2 (d). 
A main concern influencing CA-SPAD-2 performance parameters, such as afterpulsing and PDP, is the ineffective quenching. At larger excess bias voltages, the deadtime was much larger. This could be due to the small junction capacitance of the CA-SPAD-2 and re-triggering of the SPAD by carriers, generated by an avalanche event, due to fast recharge. This effect is further explained in Jegannathan et al. [25]. This "re-triggering" leads to extended deadtimes which could saturate the detector at high counting rates. An effective active quench and recharge circuit would possibly eliminate this effect and the performance can be further improved.

The performance of the CA-SPADs can be further improved when having the control over the device process (such as doping levels, thickness). Compared to conventional SPADs, CA-SPADs can offer benefits such as a scalable pixel pitch that has an absorption region decoupled from the avalanching region, a low-capacitance $p$-n junction and swift and efficient photo-response for NIR wavelengths. The two major challenges with the CA-SPADs fabricated in the $350 \mathrm{~nm}$ CMOS technology include high breakdown voltage which makes it difficult to place circuitry close by and a high dark count rate due to the topology of the p-n junction. Both these challenges can be addressed when going to a backside illuminated CA-SPAD pixel engineered with custom doping layers. The performance parameters of the two CA-SPAD iterations are compared in Table 4.

Table 4. Performance parameters of CA-SPADs.

\begin{tabular}{ccc}
\hline Device & CA-SPAD-1 [24] & CA-SPAD-2 [25] \\
\hline CMOS process & $350 \mathrm{~nm}$ & $350 \mathrm{~nm}$ \\
Foundry, Technology & X-Fab, XO035 & X-Fab, XO035 \\
Pixel pitch, shape & $40 \mu \mathrm{m}$, square & $30 \mu \mathrm{m}$, square \\
Junction, shape & $\mathrm{N}-$ well $/ \mathrm{p}-\mathrm{epi} / \mathrm{p}^{+}$, square & $\mathrm{N}$-well/p- epi/ $\mathrm{p}^{+}$, cylindrical \\
Breakdown voltage & $51 \mathrm{~V}$ & $48 \mathrm{~V}$ \\
Excess bias voltage V Vex & $0.86 \mathrm{~V}$ & $2.5 \mathrm{~V}$ \\
Timing jitter & $370 \mathrm{ps}$ & $220 \mathrm{ps}$ \\
PDP @ 785 nm [V $\left.\mathrm{V}_{\text {ex }}\right]$ & $5.6 \%[1.1 \mathrm{~V}]$ & $11.6 \%[2.5 \mathrm{~V}]$ \\
Afterpusling probability & $5.7 \%$ & $13 \%$ \\
\hline
\end{tabular}

\section{Conclusions}

The use of current assistance in several types of photodetectors for different applications has been reviewed (overview in Table 5). Most of the given examples were based on available drain-diffusions, N\&Pwells and epilayer that are available in standard $350 \mathrm{~nm}$ CMOS for making NMOS and PMOS transistors. Advanced technology options available in finer feature-size CMOS could further enhance the detection/demodulation/sampling specifications. Custom doping profiles could also lead to far better field application topologies and improve operation even further, however at the cost of additional investment.

Table 5. Summary of current-assisted photodetectors.

\begin{tabular}{|c|c|c|c|}
\hline Device & Year & $\begin{array}{c}\text { Number of } \\
\text { Pixels (Recent) }\end{array}$ & Applications \\
\hline CAPD & 2005 & $320 \times 240[39]$ & Indirect time-of-flight imaging \\
\hline CAPS & 2015 & $32 \times 32[21]$ & $\begin{array}{l}\text { Fast time-gated imaging, } \\
\text { fluorescence lifetime imaging (FLI) }\end{array}$ \\
\hline CAP & 2019 & $1[23]$ & Optical receivers \\
\hline CA-SPAD & 2019 & $1[25]$ & $\begin{array}{l}\text { Direct time-of-flight imaging, } \\
\text { low-light imaging, FLI }\end{array}$ \\
\hline
\end{tabular}

Author Contributions: Conceptualization, G.J. and M.K.; writing-original draft preparation, G.J., V.S., T.V.d.D., S.B., H.I. and M.K.; writing-review and editing, G.J., V.S., T.V.d.D., T.L., S.B., H.I. and M.K.; supervision, M.K.; project administration, M.K.; funding acquisition, M.K. All authors have read and agreed to the published version of the manuscript. 
Funding: This research was by the Research council of the Vrije Universiteit Brussel (SRP19) and the Research Foundation-Flanders (FWOAL920).

Institutional Review Board Statement: Not applicable.

Informed Consent Statement: Not applicable.

Data Availability Statement: The data presented in this study are available on request from the corresponding author.

Conflicts of Interest: The authors declare no conflict of interest.

\section{References}

1. Van Nieuwenhove, D.; van der Tempel, W.; Kuijk, M. Novel standard detector using majority current for guiding photo-generated electrons towards detecting junctions. In Proceedings of the 10th Annual Symposium IEEE/LEOS Benelux Chapter, Mons, Belgium, 1-2 December 2005; pp. 229-232.

2. van Nieuwenhove, D.; van der Tempel, W.; Grootjans, R.; Kuijk, M. Time-of-flight optical ranging sensor based on a current assisted photonic demodulator. In Proceedings of the 11th Annual Symposium IEEE/LEOS Benelux Chapter, Eindhoven, The Netherlands, 30 November-1 December 2006.

3. Hossain, Q.D.; Betta, G.F.D.; Pancheri, L.; Stoppa, D. A 3D image sensor based on current assisted photonic mixing demodulator in $0.18 \mu \mathrm{m}$ CMOS technology. In Proceedings of the 6th Conference on Ph.D. Research in Microelectronics and Electronics: PRIME 2010, Berlin, Germany, 18-21 July 2010.

4. Dalla Betta, G.F.; Donati, S.; Hossain, Q.D.; Martini, G.; Pancheri, L.; Saguatti, D.; Stoppa, D.; Verzellesi, G. Design and characterization of current-assisted photonic demodulators in 0.18- $\mu \mathrm{m}$ CMOS technology. IEEE Trans. Electron Devices 2011, 58, 1702-1709. [CrossRef]

5. Dalla Betta, G.F.; Donati, S.; Hossain, Q.D.; Martini, G.; Pancheri, L.; Stoppa, D.; Verzellesi, G. TOF-range image sensor in 0.18 um CMOS technology based on current assisted photonic demodulators. In Proceedings of the CLEO: Science and Innovations 2011, Baltimore, MD, USA, 1-6 May 2011; p. CMG6.

6. Kato, Y.; Sano, T.; Moriyama, Y.; Maeda, S.; Yamazaki, T.; Nose, A.; Shina, K.; Yasu, Y.; Van Der Tempel, W.; Ercan, A.; et al. $320 \times 240$ Back-illuminated $10 \mu \mathrm{m}$ CAPD pixels for high speed modulation Time-of-Flight CMOS image sensor. In Proceedings of the 2017 Symposium on VLSI Circuits, Kyoto, Japan, 5-8 June 2017.

7. Estrada, C.J.; Xu, C.; Chan, M. Design of current-assisted photonic demodulator (capd) for time-of-flight cmos image sensor. In Proceedings of the 2019 IEEE 13th International Conference on ASIC (ASICON), Chongqing, China, 29 October-1 November 2019; pp. 1-4.

8. Estrada, C.J.; Xiao, Y.; Xu, C.; Chan, M. Physical model of current-assisted photonic demodulator (CAPD) for time-of-flight CMOS image sensor. IEEE Trans. Electron Devices 2020, 67, 2825-2830. [CrossRef]

9. Assaf, M.; Harel, O.; Tadmor, E.; Yadid-Pecht, O.; Fish, A. Weight based current assisted photonic demodulator (WBCAPD)Expansion towards neuromorphic applications. In Proceedings of the 2020 IEEE International Symposium on Circuits and Systems (ISCAS), Virtual, 10-21 October 2020; pp. 1-5.

10. Estrada, C.J.; Xiao, Y.; Chan, M. Design considerations for current-assisted photonic demodulator (CAPD) in time-of-flight CMOS image sensor. In Proceedings of the 2020 International Symposium on VLSI Technology, Systems and Applications (VLSI-TSA), Virtual, 14 August-13 September 2020; pp. 54-55.

11. van der Tempel, W.; van Nieuwenhove, D.; Grootjans, R.; Kuijk, M. An active demodulating pixel using a current assisted photonic demodulator implemented in $0.6 \mu \mathrm{m}$ standard CMOS. In Proceedings of the 3rd IEEE International Conference on Group IV Photonics GFP, Ottawa, ON, Canada, 13-15 September 2006.

12. van Nieuwenhove, D.; van der Tempel, W.; Grootjans, R.; Stiens, J.; Kuijk, M. Photonic demodulator with sensitivity control. IEEE Sens. J. 2007, 7, 317-318. [CrossRef]

13. van der Tempel, W.; van Nieuwenhove, D.; Grootjans, R.; Kuijk, M. Lock-in pixel using a current-assisted photonic demodulator implemented in $0.6 \mu \mathrm{m}$ standard complemetary metal-oxide-semiconductor. Jpn. J. Appl. Phys. Part 1 Regul. Pap. Short Notes 2007, 46, 2377. [CrossRef]

14. van der Tempel, W.; van Nieuwenhove, D.; Grootjans, R.; Kuijk, M. Towards smarter ranging pixels with high dynamic range: Sensitivity-tuning of current assisted photonic demodulators. In Proceedings of the 2007 International Image Sensor Workshop, Ogunquit, ME, USA, 7-10 June 2007; pp. 113-116.

15. van Nieuwenhove, D.; van der Tempel, W.; Grootjans, R.; Kuijk, M. A CAPD based time-of-flight ranging pixel with wide dynamic range. In Proceedings of the Optical and Digital Image Processing, Strasbourg, France, 7-11 April 2008; Volume 7000, p. $70000 \mathrm{~N}$.

16. van der Tempel, W.; Grootjans, R.; van Nieuwenhove, D.; Kuijk, M. A 1k-pixel 3D CMOS sensor. In Proceedings of the IEEE Sensors 2008, Lecce, Italy, 26-29 October 2008; pp. 1000-1003.

17. Pancheri, L.; Stoppa, D.; Massari, N.; Malfatti, M.; Piemonte, C.; Betta, G.-F.D. Current assisted photonic mixing devices fabricated on high resistivity silicon. In Proceedings of the IEEE Sensors 2008, Lecce, Italy, 26-29 October 2008; pp. 981-983. 
18. Hossain, Q.D.; Betta, G.-F.D.; Pancheri, L.; Stoppa, D. Current assisted photonic mixing demodulator implemented in 0.18 um standard CMOS technology. In Proceedings of the 5th Conference on Ph.D. Research in Microelectronics and Electronics: PRIME 2009, Cork, Ireland, 12-17 July 2009; pp. 212-215.

19. Ingelberts, H.; Kuijk, M. High-speed gated CMOS detector for fluorescence lifetime microscopy extending to near-infrared wavelengths. In Proceedings of the IEEE Sensors 2015, Busan, Korea, 1-4 November 2015; pp. 1-4.

20. Ingelberts, H. Efficient CMOS Sensors for Sub-Nanosecond Gated Fluorescence Lifetime Imaging; Vrije Universiteit Brussel: Brussels, Belgium, 2017.

21. Ingelberts, H.; Lapauw, T.; Debie, P.; Hernot, S.; Kuijk, M. A proof-of-concept fluorescence lifetime camera based on a novel gated image sensor for fluorescence-guided surgery. In Proceedings of the Molecular-Guided Surgery: Molecules, Devices, and Applications V, San Francisco, CA, USA, 2-7 February 2019; Volume 10862, p. 12.

22. Lapauw, T.; Ingelberts, H.; Dries, T.V.D.; Kuijk, M. Sub-nanosecond time-gated camera based on a novel current-assisted CMOS image sensor. In Proceedings of the Photonic Instrumentation Engineering VI, San Francisco, CA, USA, 2-7 February 2019; Volume 10925, p. 1092506.

23. Boulanger, S.; Ingelberts, H.; Dries, T.V.D.; Gasser, A.; Kuijk, M. A novel 350 nm CMOS optical receiver based on a current-assisted photodiode detector. In Proceedings of the Silicon Photonics XIV, San Francisco, CA, USA, 2-7 February 2019; Volume 10923, p. 109231.

24. Jegannathan, G.; Ingelberts, H.; Kuijk, M. Current-assisted single photon avalanche diode (CASPAD) fabricated in $350 \mathrm{~nm}$ conventional CMOS. Appl. Sci. 2020, 10, 2155. [CrossRef]

25. Jegannathan, G.; Dries, T.V.D.; Kuijk, M. Current-assisted SPAD with improved p-n junction and enhanced NIR performance. Sensors 2020, 20, 7105. [CrossRef]

26. Sze, S.M.; Ng, K.K. Physics of Semiconductor Devices; John Wiley \& Sons: Hoboken, NJ, USA, 2006.

27. Green, M.A.; Keevers, M.J. Optical properties of intrinsic silicon at 300 K. Prog. Photovolt. Res. Appl. 1995, 3, 189-192. [CrossRef]

28. Levinshtein, M.; Rumyantsev, S.; Shur, M. Handbook Series on Semiconductor Parameters; World Scientific: Singapore, $1996 ;$ Volume 1.

29. TCAD—Silvaco. Available online: https:/ / silvaco.com/tcad/ (accessed on 16 June 2021).

30. Hout, S.I. Minority carrier accumulation at high-low junctions. Solid State Electron. 1993, 36, 1135-1142. [CrossRef]

31. Dan, Y.; Zhao, X.; Chen, K.; Mesli, A. A Photoconductor intrinsically has no gain. ACS Photon. 2018, 5, 4111-4116. [CrossRef]

32. Boulanger, S. Use of Current-Assisted Principles in Optical Receivers; Vrije Universiteit Brussel: Brussels, Belgium, 2020.

33. van der Tempel, W. Current-Assisted Sensor Devices for 3D Time-of-Flight Imaging; Vrije Universiteit Brussel: Brussels, Belgium, 2011.

34. Dicke, R.H.; Beringer, R.; Kyhl, R.L.; Vane, A.B. Atmospheric absorption measurements with a microwave radiometer. Phys. Rev. 1946, 70, 340-348. [CrossRef]

35. Ott, A. Indirect Time of Flight Range Calculation Apparatus and Method of Calculating a Phase Angle in Accordance with an Indirect Tima of Flight Range Caclulation Technique. U.S. Patent No. EP3796047A1, 20 September 2019.

36. Ott, A. Optical Range Calculation Apparatus and Method of Range Calculation. U.S. Patent No. 20192948.6, 10 December 2019.

37. Automotive Gen 2 QVGA MLX75024 Time-of-Flight (ToF) Sensor IC \#Melexis. Available online: https:/ /www.melexis.com/en/ product/MLX75024/Gen-2-QVGA-Tof-Sensor (accessed on 1 April 2021).

38. Deal, B.E.; Sklar, M.; Grove, A.S.; Snow, E.H. Characteristics of the surface-state charge (Qss) of thermally oxidized silicon. J. Electrochem. Soc. 1967, 114, 266-274. [CrossRef]

39. Kato, Y.; Sano, T.; Moriyama, Y.; Maeda, S.; Yamazaki, T.; Nose, A.; Sukegawa, S. $320 \times 240$ back-illuminated 10- $\mu$ m CAPD pixels for high-speed modulation time-of-flight CMOS image sensor. IEEE J. Solid State Circuits 2018, 53, 1071-1078. [CrossRef]

40. Kuijk, M.; Seliuchenko, V. Method and System for Demodulating Signals. U.S. Patent WO2012076500A1, 6 December 2012.

41. Papkovsky, D.B.; Dmitriev, R.I. Imaging of oxygen and hypoxia in cell and tissue samples. Cell. Mol. Life Sci. 2018, 75, 2963-2980. [CrossRef] [PubMed]

42. Becker, W. Fluorescence lifetime imaging-Techniques and applications. J. Microsc. 2012, 247, 119-136. [CrossRef] [PubMed]

43. Shimolina, L.E.; Izquierdo, M.A.; Lopez-Duarte, I.; Bull, J.A.; Shirmanova, M.V.; Klapshina, L.G.; Zagaynova, E.V.; Kuimova, M.K. Imaging tumor microscopic viscosity in vivo using molecular rotors. Sci. Rep. 2017, 7, srep41097. [CrossRef] [PubMed]

44. La Vision PicoStar HR. Available online: http:/ / www.tautec.com/LAVISION/A03\%20FL\%20PicoStar\%20HR.pdf (accessed on 1 April 2021).

45. Andor iStar U. Available online: https://andor.oxinst.com/assets/uploads/products/andor/documents/andor-istar-ccdspectroscopy-specifications.pdf (accessed on 1 April 2021).

46. PI-MAX 4 I Teledyne Princeton Instruments. Available online: https://www.princetoninstruments.com/products/pi-maxfamily/pi-max (accessed on 1 April 2021).

47. Morimoto, K.; Ardelean, A.; Wu, M.-L.; Ulku, A.C.; Antolovic, I.M.; Bruschini, C.; Charbon, E. Megapixel time-gated SPAD image sensor for 2D and 3D imaging applications. Optica 2020, 7, 346. [CrossRef]

48. Ulku, A.C.; Ardelean, A.; Antolovic, I.M.; Weiss, S.; Charbon, E.; Bruschini, C.; Michalet, X. Wide-field time-gated SPAD imager for phasor-based FLIM applications. Methods Appl. Fluoresc. 2020, 8, 024002. [CrossRef]

49. Charbon, E.; Fishburn, M.W.; Walker, R.; Henderson, R.K.; Niclass, C. SPAD-based sensors. In TOF Range-Imaging Cameras; Springer Science and Business Media LLC: Berlin/Heidelberg, Germany, 2013; pp. 11-38.

50. Bruschini, C.; Homulle, H.; Antolovic, I.M.; Burri, S.; Charbon, E. Single-photon avalanche diode imagers in biophotonics: Review and outlook. Light. Sci. Appl. 2019, 8, 1-28. [CrossRef] 
51. Gersbach, M.; Trimananda, R.; Maruyama, Y.; Fishburn, M.W.; Stoppa, D.; Richardson, J.; Walker, R.; Henderson, R.; Charbon, E. High frame-rate TCSPC-FLIM using a novel SPAD-based image sensor. In Proceedings of the Detectors and Imaging Devices: Infrared, Focal Plane, Single Photon, San Diego, CA, USA, 1-5 August 2010; Volume 7780, p. 77801H.

52. Cova, S.; Longoni, A.; Andreoni, A.; Cubeddu, R. A semiconductor detector for measuring ultraweak fluorescence decays with 70 ps FWHM resolution. IEEE J. Quantum Electron. 1983, 19, 630-634. [CrossRef]

53. Hutchings, S.W.; Johnston, N.; Gyongy, I.; Al Abbas, T.; Dutton, N.A.W.; Tyler, M.; Chan, S.; Leach, J.; Henderson, R.K. A Reconfigurable 3-D-stacked SPAD imager with in-pixel histogramming for flash LIDAR or High-speed time-of-flight imaging. IEEE J. Solid State Circuits 2019, 54, 2947-2956. [CrossRef]

54. Sze, S.; Gibbons, G. Effect of junction curvature on breakdown voltage in semiconductors. Solid Sstate Electron. 1966, 9, 831-845. [CrossRef]

55. Speeney, D.; Carey, G. Experimental study of the effect of junction curvature on breakdown voltage in Si. Solid State Electron. 1967, 10, 177-182. [CrossRef]

56. Basavanagoud, C.; Bhat, K. Effect of lateral curvature on the breakdown voltage of planar diodes. IEEE Electron Device Lett. 1985, 6, 276-278. [CrossRef] 